\title{
Modal and stability analysis of structures in periodic elastic states: application to the Ziegler column
}

\author{
Barend Bentvelsen • Arnaud Lazarus
}

Received: date / Accepted: date

\begin{abstract}
We present a spectral method to compute the transverse vibrational modes, or Floquet Forms (FFs), of a $2 D$ bi-articulated bar in periodic elastic state due to an end harmonic compressive force. By changing the directional nature of the applied load, the trivial straight Ziegler column exhibits the classic instabilities of stationary states of dynamical system. We use this simple structure as a numerical benchmark to compare the various spectral methods that consist in computing the FFs from the spectrum of a truncated Hill matrix. We show the necessity of sorting this spectrum and the benefit of computing the fundamental FFs that converge faster. Those FFs are almost-periodic entities that generalize the concept of harmonic modal analysis of structures in equilibria to structures in periodic states. Like their particular harmonic relatives, FFs allow to get physical insights in the bifurcations of periodic stationary states. Notably, the local loss of stability is due to the frequency lock-in of the FFs for certain modulation parameters. The presented results could apply to many structural problems in mechanics, from the vibrations of rotating machineries with shape imperfections to the stability of periodic limit cycles or of any slender structures with tensile or compressive periodic elastic stresses.
\end{abstract}

Keywords Floquet theory · Modal analysis · Stability analysis - Structural mechanics - Bifurcation analysis

B. Bentvelsen · A. Lazarus

Sorbonne Universités, UPMC Univ Paris 06, CNRS, UMR 7190, Institut Jean Le Rond d'Alembert, F-75005, Paris, France

E-mail: arnaud.lazarus@upmc.fr

\section{Introduction}

Modal analysis is a key concept in the study of mechanical vibrations that is today commonly used by scientists and engineers in various fields from molecular chemistry [36] to aerospace industries [10]. It is a spectral numerical method consisting in decomposing the first order perturbation around an equilibrium state of a dynamical system in a linear superposition of harmonic eigenmodes [13]. In practice, those modes allow to reveal intrinsic vibrational properties [28] or the local stability behavior of structures in equilibria $[21,31]$. They can also be used as a projection basis to reduce the dimensionality of linear or nonlinear vibrational structural models [23,25].

Thanks to Floquet theory $[11,44]$, it should be possible to generalize this modal approach to dynamical systems in periodic stationary states, i.e. states whose properties vary periodically with time [4]. In structural dynamics, this includes rotating machineries with imperfections [12] or structures submitted to periodic compression or tension axial loadings such as slender beams or plates undergoing large vibrations [29]. Mathematically, a perturbation around a periodic state can be decomposed in a linear surperposition of almost periodic eigenfunctions, called Floquet forms (FFs). Like vibrational modes for an equilibrium, FFs could reveal the intrinsic vibrational properties of a structure in periodic state and determine its local stability. Floquet theory is numerically applied in periodically time-varying linear systems [4] or nonlinear dynamical systems [14,30] but the spectral computation and physical meaning of FFs have been overlooked.

In the time domain, two main families of numerical methods exist that derive from Floquet theory. One possibility is to compute the Monodromy or state tran- 
sition matrix and its eigenvalues (Floquet multipliers) that assess the local stability of the perturbed periodic stationary state $[33,43]$. Although it may be possible to recover FFs from the eigenvectors of this matrix, they are usually ignored. A second technique consists in using the Lyapunov-Floquet transformation to recast a linear time-periodic system in a time-invariant one $[37,38]$. This transformation could allow to compute and analyze FFs but it has mostly been used as a step, which coupled with center manifold reduction techniques and normal form theories, enables the study of nonlinear time-periodic systems undergoing bifurcations $[5,32,39]$.

In the frequency domain, the calculation of the spectrum of Hill's matrix [17] associated with the perturbed periodic state should theoretically give direct access to FFs, but the computational reality is quite different as upon numerical truncation, the convergence of Hill's matrix is subjected to controversy $[6,34,35]$. As a consequence, two main approaches have been developed to apply Floquet theory. The first numerical approach consists in imposing particular fundamental frequencies of FFs, or Floquet exponents, to maintain the selfadjointness of the original perturbed Hill problem and discard any spurious spectrum. Whether it is marginal boundary Floquet exponents associated with harmonic and subharmonic FFs $[1,19]$ or intermediate ones varied within the reciprocal primitive lattice of a Bloch wave analysis $[7,8,18]$, those methods compute the parameters of the periodic state associated with a given Floquet mode. This is different from modal analysis that would supposedly seek for the FFs associated with a given perturbed stationary state. The latter could be done by directly computing the truncated spectrum of Hill's matrix for a given periodic state. But a confusion subsists as for the sorting method one should use for the spurious computed spectrum, between eigenvalue $[9,27,45]$ or eigenvector sorting $[2,22,26,41]$. Furthermore, only Floquet exponents are usually considered and modal informations from FFs are usually neglected. Floquet modes have been computed for the linear vibration analysis of non axisymmetric rotating machineries such as cracked or geometrically imperfect rotors but the computations are based on Hill's truncated matrix without sorting algorithm $[15,16,20,24]$, a method that is known to give erroneous results in some cases, especially when dealing with resonant nonlinear dynamical systems.

Here, we show how to compute FFs in the spectral domain and highlight their potential for modal analysis of structures in periodic states, through a simple benchmark model: the Ziegler column [3,42]. The discrete dynamical system we consider is a classic $2 D$ model consisting of two articulated rigid bars, connected by elastic hinges, that are submitted to an end periodic compressive load so that the elastic state of the Ziegler column is periodically modulated [32]. We focus on the transverse oscillatory modes of the structure around its trivial configuration that is the undeformed straight column in space, with periodic elasticity in time.

In Section 2, we describe the nonlinear equations of motion of our model as well as the time-varying linear ordinary differential equations describing the transverse linear oscillations about the trivial state. By considering a non-conservative following or conservative horizontal compressive loading, we show that our benchmark model captures the classic bifurcations and local instabilities of fixed points and periodic states of discrete dynamical systems. We then recall the Floquet-Hill frequency method and review the various spectral sorting techniques that exist to compute FFs. In Section 3, we perform the modal and stability analysis of the periodically conservative case. In Section 4, we investigate the influence of a non-conservative positional loading on the computation of FFs and their associated stability. In both periodically conservative and non-conservative cases, we compare the efficiency of the spectral sorting methods for computing FFs. All our stability results are validated through the Monodromy matrix algorithm.

Like for classic modal analysis, the free transverse vibration of a structure in periodic elastic state can be decomposed in a linear combination of its FFs. As classic modes are constant eigenfunctions, harmonically vibrating; FFs are periodic eigenfunctions, harmonically modulated. Computation of FFs is crucial since the loss of local stability is due to frequency lock-in of FFs in the parameter space of the periodic state. In the conservative case, the Floquet modes are uncoupled. Period doubling and stationary bifurcations are explained by the frequency lock-in of a Floquet mode and its conjugate in the state space. This mechanism is a generalization of the buckling of an equilibrium configuration seen through its vibrational harmonic modes. In the non-conservative case, the computed FFs are coupled. Secondary Hopf bifurcations are explained by the frequency lock-in between two physical FFs in the parameter space of the periodic state. Similarly, Hopf bifurcation is explained by a frequency lock-in between two classic modes. Finally, we show that not sorting the spectrum of Hill's truncated matrix leads to erroneous stability results. We also highlight the fact that sorting the eigenvectors instead of the eigenvalues of the Hill's matrix converge faster in the non-conservative case and for slow modulation in the conservative case.

Our results give new physical insights on the natural relation between classic harmonic modes of vibrations 
and Floquet modes for structures that are in periodic elastic states. This paper also clarifies the debate about the Floquet-Hill frequency method to assess the stability of periodic states by clearly showing the necessity of sorting the spectral outcomes of the Hill matrix. Those results could pave the way for a modal analysis of structures in periodic states and the use of Floquet forms for stability analysis, structural design, or possible candidates for modal reduction techniques.

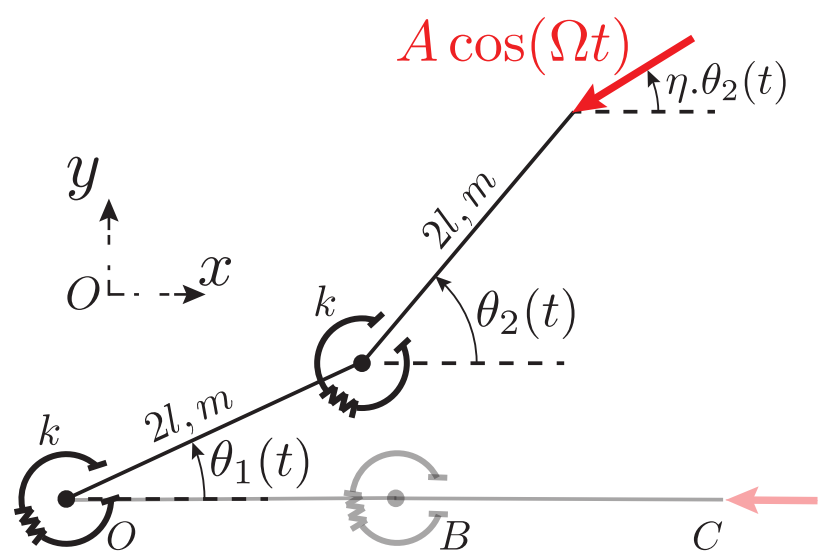

Fig. 1 The $2 D$ structure under study is a bi-articulated bar submitted to a compressive periodic load at its end either conservative $(\eta=0$, i.e. horizontal force) or non conservative $(\eta=1$, i.e. following force).

\section{Nonlinear and linearized equations of motion of the Ziegler column in periodic elastic state}

\subsection{Nonlinear equation of motion}

To illustrate the concept of Floquet forms, we consider the archetypal example of the planar bi-articulated bar illustrated in Fig. 1, also known as the Ziegler column $[32,40,42]$. The rigid and inextensible bars of length $2 l$ have a mass $m$. The two bars are allowed to rotate at points $O$ and $B$ thanks to elastic hinges characterized by a rotational stiffness $k$. At rest, the biarticulated structure is lying in the horizontal direction $(O, x)$. The structure is possibly moving in the plane $(O, x, y)$ under the action of a $T$-periodic end compressive force $P(t)=P(t+T)=A \cos (\Omega t)$ with fundamental frequency $\Omega=2 \pi / T$. This force is either periodically conservative $(\eta=0$, i.e. horizontal force) or non-conservative $(\eta=1$, i.e. following force). The motion of this structure is completely parameterized by the two angles $\theta_{1}(t)$ and $\theta_{2}(t)$ between the horizontal axis $(O, x)$ and the first and second bar, respectively. In this paper, we are interested in the linear vibrations of this two degrees-of-freedom structure about the trivial straight state $\theta_{1}(t)=\theta_{2}(t)=0$. Such a model is qualitatively representative of a tremendous amount of applications in structural mechanics as it exhibits most of the classic bifurcations of dynamical systems, although the perturbed stationary state is spatially trivial. Because the applied end load is possibly periodic, classic modal analysis fails to analyze such a simple system whose elasticity may vary periodically with time and Floquet modes will be needed.

Balancing the quantity of acceleration of each bar of the bi-articulated elastic system with the applied external moments (the expression of those quantities are given in Appendix 1), the nonlinear equation of motion of the Ziegler column, reads, in the physical space $\left(\theta_{1}(t), \theta_{2}(t)\right)$ :

$$
\begin{aligned}
0 & =\frac{16}{3} m l^{2} \ddot{\theta}_{1}+2 m l^{2} \ddot{\theta}_{2} \cos \left(\theta_{1}-\theta_{2}\right) \\
& +2 m l^{2} \dot{\theta}_{2}^{2} \sin \left(\theta_{1}-\theta_{2}\right)+2 k \theta_{1}-k \theta_{2} \\
& +2 l A \cos (\Omega t)\left[\cos \left(\theta_{1}\right) \sin \left(\eta \theta_{2}\right)-\sin \left(\theta_{1}\right) \cos \left(\eta \theta_{2}\right)\right] \\
0 & =\frac{4}{3} m l^{2} \ddot{\theta}_{2}+2 m l^{2} \ddot{\theta}_{1} \cos \left(\theta_{1}-\theta_{2}\right) \\
& -2 m l^{2} \dot{\theta}_{1}^{2} \sin \left(\theta_{1}-\theta_{2}\right)+k \theta_{2}-k \theta_{1} \\
& +2 l A \cos (\Omega t)\left[\cos \left(\theta_{2}\right) \sin \left(\eta \theta_{2}\right)-\sin \left(\theta_{2}\right) \cos \left(\eta \theta_{2}\right)\right]
\end{aligned}
$$

By introducing the dimensionless time variable $\tau=\omega_{n} t$ with a reference natural frequency $\omega_{n}=\sqrt{k /\left(m l^{2}\right)}$ and multiplying the first and second line of Eq.(1) by $3 /(16 k)$ and $3 /(4 k)$, respectively, one can rewrite Eq.(1) in the dimensionless form

$$
\begin{aligned}
0 & =\ddot{\theta}_{1}+\frac{3}{8} \ddot{\theta}_{2} \cos \left(\theta_{1}-\theta_{2}\right) \\
& +\frac{3}{8} \dot{\theta}_{2}^{2} \sin \left(\theta_{1}-\theta_{2}\right)+\frac{3}{8} \theta_{1}-\frac{3}{16} \theta_{2} \\
& +\lambda \cos (\beta \tau)\left[\cos \left(\theta_{1}\right) \sin \left(\eta \theta_{2}\right)-\sin \left(\theta_{1}\right) \cos \left(\eta \theta_{2}\right)\right] \\
0 & =\ddot{\theta}_{2}+\frac{3}{2} \ddot{\theta}_{1} \cos \left(\theta_{1}-\theta_{2}\right) \\
& -\frac{3}{2} \dot{\theta}_{1}^{2} \sin \left(\theta_{1}-\theta_{2}\right)+\frac{3}{4} \theta_{2}-\frac{3}{4} \theta_{1} \\
& +4 \lambda \cos (\beta \tau)\left[\cos \left(\theta_{2}\right) \sin \left(\eta \theta_{2}\right)-\sin \left(\theta_{2}\right) \cos \left(\eta \theta_{2}\right)\right]
\end{aligned}
$$

where ( ) denotes differentiation with respect to $\tau, \beta=$ $\Omega / \omega_{n}$ is the ratio between the excitation and the reference natural frequency and $\lambda=A /(8 k / 3 L)$ represents the dimensionless amplitude of the harmonic compressive load. The dimensionless period of the applied compressive force now reads $\bar{T}=2 \pi / \beta$.

Eq.(2) is an implicit nonlinear system of Ordinary Differential Equations (ODEs) with periodic coefficients in the form $\mathbf{f}(\mathbf{x}(t), \dot{\mathbf{x}}(t), t)=,\mathbf{0}$, where the 4-dimensional state vector reads $\mathbf{x}(t)=\left\{\theta_{1}, \theta_{2}, \dot{\theta}_{1}, \dot{\theta}_{2}\right\}^{T}$. By changing the dimensionless loading parameters $\lambda$ and $\beta$ in the 

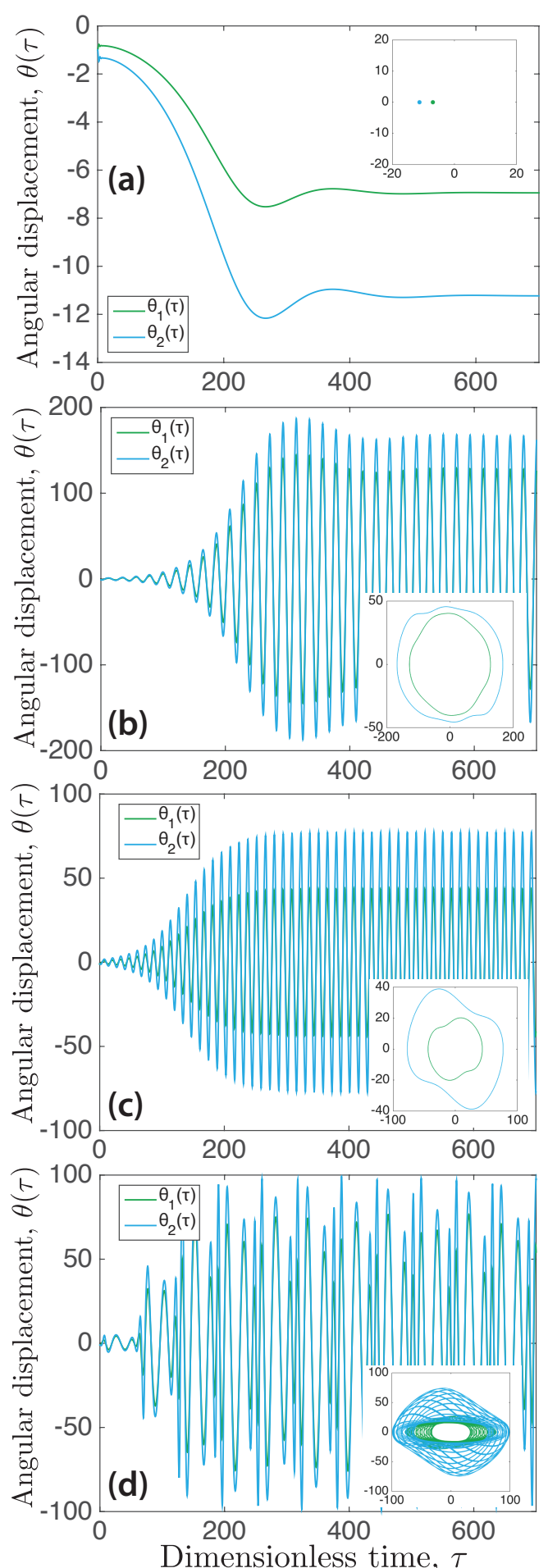

Fig. 2 Nonlinear dynamical response for various loading parameters and initial conditions $\theta_{1}(0)=\theta_{2}(0)=-1^{\circ}$ and $\dot{\theta}_{1}(0)=\dot{\theta}_{2}(0)=0$. A little amount of viscous damping has been introduced to help the simulations. (a) Bifurcation to an equilibrium state for $\eta=0, \beta=0$ and $\lambda=0.072$. (b) Flip bifurcation to a dynamic state with a $2 \bar{T}$-period for $\eta=0$, $\beta=0.584$ and $\lambda=0.036$. (c) Hopf bifurcation to a periodic stationary state for $\eta=1, \beta=0$ and $\lambda=0.4$. (d) Secondary Hopf or Neimark-Sacker bifurcation on a quasi-periodic state for $\eta=1, \beta=0.1$ and $\lambda=0.6$. Insets show the bifurcated stationary states in the state space $(\theta(\tau), \dot{\theta}(\tau))$. conservative case $\eta=0$ or non-conservative one $\eta=1$ and for a given set of initial conditions $\mathbf{x}(0)$, this simple systems exhibits most of the qualitative vibrational behavior of stationary states of nonlinear dynamical systems as shown in Fig. 2.

2.2 Modal analysis of the trivial periodic elastic state $\theta_{1}^{0}(\tau)=\theta_{2}^{0}(\tau)=0$

To get a deeper physical understanding of the rich qualitative behavior exhibited by the Ziegler column in Fig. 2 , we study the linear vibrations around the trivial spatial state $\theta_{1}^{0}(\tau)=\theta_{2}^{0}(\tau)=0$ that verifies the dimensionless equation of motion given in Eq.(2).

Replacing $\theta_{1}(\tau)$ and $\theta_{2}(\tau)$ by their first order perturbed expressions $\theta_{1}(\tau)=\theta_{1}^{0}(\tau)+\varepsilon \theta_{1}^{*}(\tau)$ and $\theta_{2}(\tau)=$ $\theta_{2}^{0}(\tau)+\varepsilon \theta_{2}^{*}(\tau)$ in the nonlinear equation of motion Eq. $(2)$ and equating the first power of the small parameter $\varepsilon$, one obtains the linearized equation of motion in the vicinity of the considered state $\theta_{1}^{0}(\tau)=\theta_{2}^{0}(\tau)=0$,

$\mathbf{M} \ddot{\mathbf{u}}(\tau)+\mathbf{K}(\tau) \mathbf{u}(\tau)=\mathbf{0}$

where

$$
\begin{aligned}
& \mathbf{u}(\tau)=\left\{\begin{array}{l}
\theta_{1}^{*}(\tau) \\
\theta_{2}^{*}(\tau)
\end{array}\right\}, \mathbf{M}=\left[\begin{array}{ll}
1 & \frac{3}{8} \\
\frac{3}{2} & 1
\end{array}\right] \text { and } \\
& \mathbf{K}(\tau)=\left[\begin{array}{cc}
\frac{3}{8} & -\frac{3}{16} \\
-\frac{3}{4} & \frac{3}{4}
\end{array}\right]+\lambda \cos (\beta \tau)\left[\begin{array}{cc}
-1 & \eta \\
0 & 4 \eta-4
\end{array}\right]
\end{aligned}
$$

are the vector of physical degrees of freedom, the mass and stiffness matrix, respectively.

For $\beta=0$, Eq.(3) is the one of a linearized Ziegler column under constant compressive loading and quantities such as natural vibrational frequencies, critical buckling and critical flutter loads can be classically determined. By looking at the eigenvalues of the stiffness matrix for $\eta=0$, it is possible to assess the critical buckling load of the fundamental elastic state. The condition $\operatorname{det}(\mathbf{K}(\lambda))=0$ gives a critical buckling load $\lambda_{b}=(9-3 \sqrt{5}) / 32$. By analyzing the dynamical problem given in Eq.(3) for no compressive loading, $\lambda=0$, one can compute the dimensionless natural frequencies of the straight Ziegler column. The condition $\operatorname{det}(\mathbf{K}(\lambda=$ $\left.0)-\omega^{2} \mathbf{M}\right)=0$ gives us the natural frequencies of the unloaded system $\omega_{1}=((27-3 \sqrt{74}) / 14)^{1 / 2}$ and $\omega_{2}=((27+3 \sqrt{74}) / 14)^{1 / 2}$. Finally, by finding the minimal $\lambda$ for which the condition $\operatorname{det}\left(\mathbf{K}(\lambda)-\omega^{2} \mathbf{M}\right)=0$ leads to $\Re(\omega)>0$ for $\beta=0$ and $\eta=1$, we obtain $\lambda_{f}=((135 / 8)-2 \sqrt{1575 / 256}) / 25$, the critical flutter instability threshold above which the Ziegler column in constant elastic state undergoes a Hopf bifurcation [42].

Working with the 4-dimensional vector of state variables $\mathbf{y}(\tau)=\left\{\theta_{1}^{*}, \theta_{2}^{*}, \dot{\theta}_{1}^{*}, \dot{\theta}_{2}^{*}\right\}^{T}$ instead of $\mathbf{u}(\tau)$, the linear 
system of ODEs given in Eq.(3) can be recast in the generalized form

$\mathbf{B} \dot{\mathbf{y}}(\tau)-\mathbf{A}(\tau) \mathbf{y}(\tau)=\mathbf{0}$

with $\mathbf{B}=\left[\begin{array}{ll}\mathbf{0}_{2} & \mathbf{M} \\ \mathbf{M} & \mathbf{0}_{2}\end{array}\right]$ and $\mathbf{A}(\tau)=\left[\begin{array}{cc}-\mathbf{K}(\tau) & \mathbf{0}_{2} \\ \mathbf{0}_{2} & \mathbf{M}\end{array}\right]$

and where $\mathbf{0}_{n}$ is the $n \times n$ null matrix. Inverting the constant matrix $\mathbf{B}$, the structural vibrational problem of Eq.(3) can be transformed from the physical space to the dynamical state space,

$\dot{\mathbf{y}}(\tau)=\mathbf{J}(\tau) \mathbf{y}(\tau)$

with

$$
\begin{aligned}
\mathbf{J}(\tau) & =\mathbf{B}^{-1} \mathbf{A}(\tau)=\frac{1}{2} \mathbf{J}_{c}^{0}+\mathbf{J}_{c}^{1} \cos (\beta \tau) \\
& =\mathbf{J}^{0}+\mathbf{J}^{1} e^{i \beta \tau}+\mathbf{J}^{-1} e^{-i \beta \tau}, \\
\mathbf{J}^{0}= & \frac{1}{2} \mathbf{J}_{c}^{0}=\left[\begin{array}{cccc}
0 & 0 & 1 & 0 \\
0 & 0 & 0 & 1 \\
-\frac{3}{2} & \frac{15}{14} & 0 & 0 \\
3 & -\frac{33}{14} & 0 & 0
\end{array}\right]
\end{aligned}
$$

and $\mathbf{J}^{1}=\mathbf{J}^{-1}=\frac{1}{2} \mathbf{J}_{c}^{1}=\lambda\left[\begin{array}{cccc}0 & 0 & 0 & 0 \\ 0 & 0 & 0 & 0 \\ \frac{8}{7} & \left(\frac{4}{7} \eta-\frac{12}{7}\right) & 0 & 0 \\ -\frac{12}{7} & \left(-\frac{20}{7} \eta+\frac{32}{7}\right) & 0 & 0\end{array}\right]$.

One sees from the linear ODE of Eq.(5) that the timevarying Jacobian $\mathbf{J}(\tau)$ of the nonlinear equation of motion Eq.(2) evaluated at $\theta_{1}^{0}(\tau)=\theta_{2}^{0}(\tau)=0$ is $\bar{T}$-periodic with minimal period $\bar{T}=2 \pi / \beta$ in our case. Eq. (5) $\bmod -$ els the linear vibrations about the straight Ziegler column in a periodic elastic state. To study this linear ODE with periodically time-varying coefficients, we can use Floquet theory $[11,44]$.

According to Floquet theory, the $N$-dimensional linear system Eq.(5) has $N=4$ linearly independent solutions $\mathbf{y}_{n}(\tau)$, so that any solution $\mathbf{y}(\tau)$ can be written:

$\mathbf{y}(\tau)=\sum_{n=1}^{N} c_{n} \mathbf{y}_{n}(\tau)$

where $c_{n}$ are $N$ constants that depend upon the initial conditions $\mathbf{y}(0)$ and $\mathbf{y}_{n}(\tau)$ are called fundamental solutions. According to the same theory, it is possible to express the fundamental solutions $\mathbf{y}_{n}(\tau)$ in the so-called Floquet form

$\mathbf{y}_{n}(\tau)=\mathbf{p}_{n}(\tau) e^{s_{n} \tau}$

where $\mathbf{p}_{n}(\tau)=\mathbf{p}_{n}(\tau+\bar{T})$ is a $N$-dimensional complex vector function of period $\bar{T}$ and $s_{n}$ is a complex number called a Floquet exponent. Developing the unknown periodic function $\mathbf{p}_{n}(\tau)$ in a complex Fourier series $\mathbf{p}_{n}(\tau)=\sum \mathbf{p}_{n}^{h} e^{i h \beta \tau}$, the FFs in Eq.(7) can be rewritten in term of the fundamental frequency $\beta=$ $2 \pi / \bar{T}$ :

$\mathbf{y}_{n}(\tau)=\sum_{h=-\infty}^{+\infty} \mathbf{p}_{n}^{h} e^{\left(i h \beta+s_{n}\right) \tau}$

The $\mathrm{FF} \mathbf{y}_{n}(\tau)$ is an almost periodic oscillation whose spectrum depends on the periodicity $\bar{T}$ of the spatially trivial elastic state of the Ziegler column. In the particular case when $\beta=0$, the eigenfunction $\mathbf{p}(\tau)$ is constant and the normal form in Eq.(7)-(8) is a harmonic mode of vibration as it is defined in classic structural dynamics. The FFs for structures in periodic state can therefore be seen as a generalization of a vibrational mode of a structure in equilibrium.

In this work, we would like to compute the FFs of a Ziegler column for a given compressive end periodic load. One direct way to do it is to expand the $\bar{T}$-periodically time-varying Jacobian of Eq.(5) in the Fourier series

$\mathbf{J}(\tau)=\sum_{k=-\infty}^{+\infty} \mathbf{J}^{k} e^{i k \beta \tau}$.

In the particular case of a harmonic end compressive load with the Jacobian given in Eq.(5), Eq.(9) is simplified in closed form as the contributions of $\mathbf{J}(\tau)$ are limited to the fundamental and first harmonic, i.e. $\mathbf{J}^{k}=\mathbf{0}_{N}$ for $|k|>1$.

Replacing the perturbed solution $\mathbf{y}(\tau)$ by its expanded Floquet normal form Eq.(8) and the jacobian $\mathbf{J}(\tau)$ by its Fourier expansion Eq. (9) in the periodically time-varying linear ODE Eq.(5), and balancing every harmonic, we obtain an extended eigenvalue problem in the spectral domain

$\left(\mathbf{H}^{H}-s \mathbf{1}\right) \mathbf{q}^{H}=\mathbf{0}$

where, for example,

$\mathbf{H}^{2}=\left[\begin{array}{ccccc}\mathbf{J}^{0}+i 2 \beta \mathbf{1}_{4} & \mathbf{J}^{-1} & \mathbf{J}^{-2} & \mathbf{J}^{-3} & \mathbf{J}^{-4} \\ \mathbf{J}^{1} & \mathbf{J}^{0}+i \beta \mathbf{1}_{4} & \mathbf{J}^{-1} & \mathbf{J}^{-2} & \mathbf{J}^{-3} \\ \mathbf{J}^{2} & \mathbf{J}^{1} & \mathbf{J}^{0} & \mathbf{J}^{-1} & \mathbf{J}^{-2} \\ \mathbf{J}^{3} & \mathbf{J}^{2} & \mathbf{J}^{1} & \mathbf{J}^{0}-i \beta \mathbf{1}_{4} & \mathbf{J}^{-1} \\ \mathbf{J}^{4} & \mathbf{J}^{3} & \mathbf{J}^{2} & \mathbf{J}^{1} & \mathbf{J}^{0}-i 2 \beta \mathbf{1}_{4}\end{array}\right]$.

is the complex Hill matrix truncated to the order $H=$ $2, \mathbf{1}_{N}$ is the identity matrix of size $N=4, \mathbf{1}$ is the identity matrix of size $N \times(2 H+1)$ and $\mathbf{0}$ is a null vector of same dimension. More details about the derivation of the complex and real Hill matrix from the harmonic balance method can be found in Appendix 2 and Appendix 3 , respectively.

Computing the spectrum of the square Hill matrix $\mathbf{H}^{H}$ with size $N \times(2 H+1)$, we get $N \times(2 H+1)$ 
eigenvalues $s_{l}$ and complex eigenvectors $\mathbf{q}_{l}^{H}$ of size $N \times$ $(2 H+1)$. For $H=2, \mathbf{q}_{l}^{H=2}$ is in the form $\mathbf{q}_{l}^{H=2}=$ $\left\{\mathbf{p}_{l}^{-2} \mathbf{p}_{l}^{-1} \mathbf{p}_{l}^{0} \mathbf{p}_{l}^{1} \mathbf{p}_{l}^{2}\right\}^{T}$ where $\mathbf{p}_{l}^{h}$ is the harmonic contribution of the $l^{\text {th }}$ Floquet form $\mathbf{y}_{l}(\tau)$ given in Eq.(8). Replacing the computed eigenvalues $s_{l}$ and eigenvectors $\mathbf{q}_{l}^{H}$ in the expression Eq.(8), we get $N \times(2 H+1)$ numerically approximated Floquet forms $\mathbf{y}_{l}(\tau)$. Since we were theoretically waiting for $N$ Floquet forms in the state space, some information is redundant and some interpretation is needed.

When $H \rightarrow \infty$, the computed eigenvalues and eigenvectors are not all independent. Actually, there are $N$ independent families of solutions that verify the relations $s_{n+k}=s_{n}+i k \beta$ and $\mathbf{p}_{n+k}^{h+k}=\mathbf{p}_{n}^{h}$ for $n=1 \ldots N$, $-\infty<k<+\infty$ and $-\infty<h<+\infty$. By replacing the $N$ families of infinite eigenvalues and eigenvectors in Eq.(8), only $N$ Floquet forms $\mathbf{y}_{n}(\tau)$ are obtained, the rest of the information being redundant. In practice, when truncating $H$ to a finite value, only some of the $N \times(2 H+1)$ computed eigenvalues $s_{l}$ and eigenvectors $\mathbf{q}_{l}^{H}$ eventually converge, as $H$ is increased, to the aforementioned relations; some of the spectrum is spurious, whatever the chosen truncation order. The presence of this spurious spectrum lies in the fact that the infinite Hill operator $\mathbf{H}^{\infty}$ is self-adjoint when the truncated one $\mathbf{H}^{H}$ is not (a short explanation is given at the end of Appendix 3). As a consequence, there is a necessity to sort the computed spectrum to obtain the $N$ converged Floquet forms. Based on the particular relations between eigenvalues or eigenvectors that exist for $H \rightarrow \infty$, two different sorting strategies can be used:

1. Eigenvalue sorting: For the converged part of the computed spectrum, we have the particular relations $s_{n+k}=s_{n}+i k \beta$ for $n=1 \ldots N$ and $-H \leq$ $k \leq+H$. By taking the $N$ eigenvalues whose imaginary parts are contained in the spectral primitive cell $-\beta / 2 \leq \Im\left(s_{l}\right)<\beta / 2$, the latter will eventually converge to Floquet exponents as $H$ is increased. Replacing those $N$ converged eigenvalues $s_{l}$ with their associated eigenvectors $\mathbf{q}_{l}^{H}$ in Eq.(8), we can reconstruct the $N$ Floquet forms $\mathbf{y}_{n}(\tau)$. The convergence of the sorted eigenvalues has been rigorously proved [27,45].

2. Eigenvector sorting: Since the converged eigenvectors verify $\mathbf{p}_{n+k}^{h+k}=\mathbf{p}_{n}^{h}$ for $-H \leq k \leq+H$ and $-H \leq h \leq+H$, the $N$ fundamental eigenvectors $\mathbf{q}_{n}^{H}$ associated with the fundamental Floquet exponents $s_{n+k}=s_{n}+i k \beta$ for $k=0$, are the most symmetric ones as compared to $\mathbf{p}_{n}^{0}[22]$. To compute those $N$ fundamental eigenvectors in practice, we compute the $N \times(2 H+1)$ weighted means $w_{l}=$ $\sum_{h} h\left|\mathbf{p}_{l}^{h}\right| / \sum_{h}\left|\mathbf{p}_{l}^{h}\right|$. In this dual space, the converged
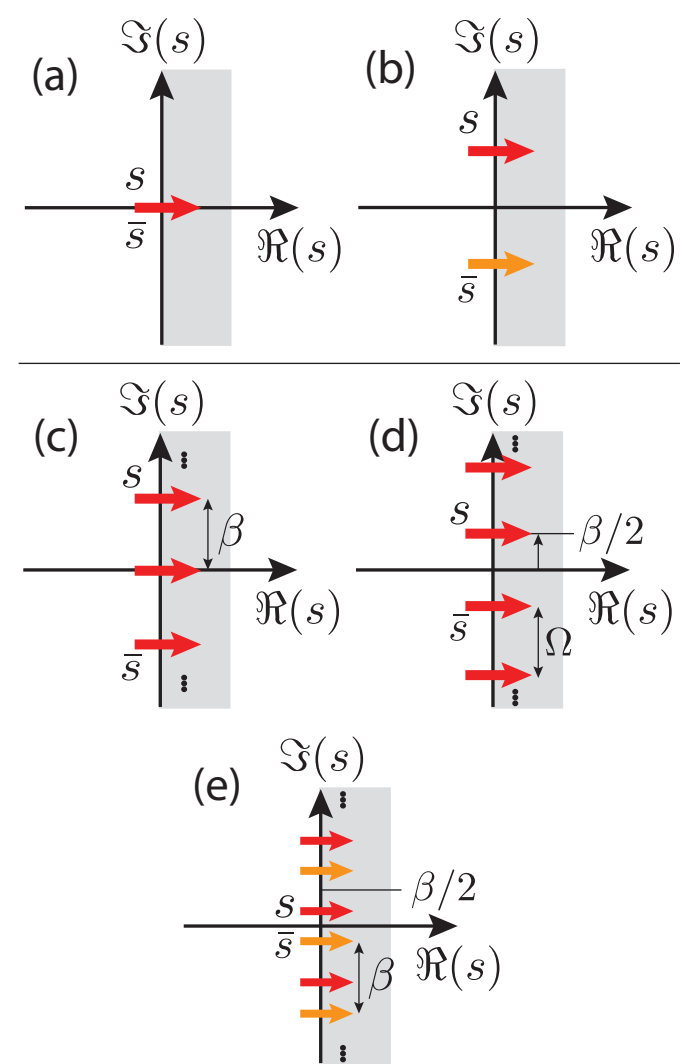

Fig. 3 Bifurcation and local stability analysis of a periodic stationary state by studying the spectrum of the destabilizing Floquet form in the Argand plane. (a) Static instability leading to a steady-state bifurcation. (b) Dynamical instability responsible for the Hopf bifurcation. (c) Steady bifurcation of a $T$-periodic state. (d) Flip or period doubling bifurcation of a periodic state. (e) Secondary Hopf or Neimark-Sacker bifurcation of a periodic state.

spectrum verifies $w_{n+k}=w_{n}+k$. The $N$ eigenvectors and associated eigenvalues that lead to the $N$ fundamental Floquet forms $\mathbf{y}_{n}(\tau)$ through Eq.(8), are the ones inside the primitive cell $-1 / 2 \leq w_{l}<$ $1 / 2$. Currently, there is no mathematical proof on the convergence of this sorting method but we will show that computing the $N$ fundamental FFs associated with $k=0$ is often more efficient than the eigenvalue sorting method, especially for small fundamental frequency of the periodic state $\beta$.

Like a vibrational mode for a perturbed equilibrium, the $N$ computed FFs $\mathbf{y}_{n}(\tau)$, with complex spectrum $\sum_{h}\left(s_{n}+i h \beta\right)$, allow to determine the local stability of a perturbed periodic stationary state. Notably, if it exists a subscript $g$ for which $\Re\left(s_{g}\right)>0$, the perturbed stationary state increases exponentially in the direction of the $g^{t h}$ mode $\mathbf{y}_{g}(\tau)$ and the stationary state is said to be locally unstable. By analyzing how the spectrum $\sum_{h}\left(s_{g}+i h \beta\right)$ and its complex conjugate in the state space $\sum_{h}\left(\bar{s}_{g}+i h \beta\right)$ cross the imaginary axis 
in the Argand plane, as shown in Fig. 3, it is possible to characterize the bifurcation that will undergo the perturbed stationary state. Note that for both algorithms, it is important to exclude one of the limit of the ranges $-\beta / 2 \leq \Im\left(s_{l}\right)<\beta / 2$ or $-1 / 2 \leq w_{l}<1 / 2$ in order to keep $N$ eigenvalues or eigenvectors even if the conjugate spectra lock in $i(\beta / 2 \pm m \beta)$ as shown in Fig. 3(d).

In the following, we compare and challenge the aforementioned algorithms by computing the FFs and local stability of the Ziegler column in the straight trivial spatial state and periodic elastic state as shown in Fig. 1. In practice, this is done by computing and sorting the spectrum of the truncated Hill matrix $\mathbf{H}^{H}$ of Eq.(10) derived from the Jacobian $\mathbf{J}(\tau)$ given in Eq.(5). Section 3 concerns the case of a conservative compressive end force with $\eta=0$ where only the bifurcations of Figs. $3(\mathrm{a})$,(c) and (d) can be observed. Section 4 deals with a nonconservative following end compressive load, i.e. for $\eta=1$, where Hopf bifurcations as illustrated in Figs. 3(b) and (e) can happen as well.

\section{Periodically conservative case $(\eta=0)$}

In this section, we analyze the influence of the modulation loading parameters $\beta$ and $\lambda$ on the transverse vibrational modes and stability of the Ziegler column under an end compressive horizontal load $(\eta=0)$. We start with the classic case of a constant compressive force, i.e. for $\beta=0$ and study the influence of periodicity when $\beta \neq 0$ on the harmonic modes and stability. We finish with some remarks on the particular limit $\beta \rightarrow 0$.

\subsection{Constant elastic state $(\beta=0)$}

When $\beta=0$, the applied compressive dimensionless load $\bar{P}(\tau)=\lambda \cos (\beta \tau)$ is constant in time and the Hill matrix of Eq.(10) truncated to the order $H=2$ becomes

$$
\mathbf{H}^{2}=\left[\begin{array}{lllll}
\mathbf{J}^{0} & \mathbf{0}_{4} & \mathbf{0}_{4} & \mathbf{0}_{4} & \mathbf{0}_{4} \\
\mathbf{0}_{4} & \mathbf{J}^{0} & \mathbf{0}_{4} & \mathbf{0}_{4} & \mathbf{0}_{4} \\
\mathbf{0}_{4} & \mathbf{0}_{4} & \mathbf{J}^{0} & \mathbf{0}_{4} & \mathbf{0}_{4} \\
\mathbf{0}_{4} & \mathbf{0}_{4} & \mathbf{0}_{4} & \mathbf{J}^{0} & \mathbf{0}_{4} \\
\mathbf{0}_{4} & \mathbf{0}_{4} & \mathbf{0}_{4} & \mathbf{0}_{4} & \mathbf{J}^{0}
\end{array}\right]
$$

with

$$
\mathbf{J}^{0}=\left[\begin{array}{cccc}
0 & 0 & 1 & 0 \\
0 & 0 & 0 & 1 \\
-\frac{3}{2} & \frac{15}{14} & 0 & 0 \\
3 & -\frac{33}{14} & 0 & 0
\end{array}\right]+\lambda\left[\begin{array}{cccc}
0 & 0 & 0 & 0 \\
0 & 0 & 0 & 0 \\
\frac{8}{7} & -\frac{12}{7} & 0 & 0 \\
-\frac{12}{7} & \frac{32}{7} & 0 & 0
\end{array}\right] .
$$
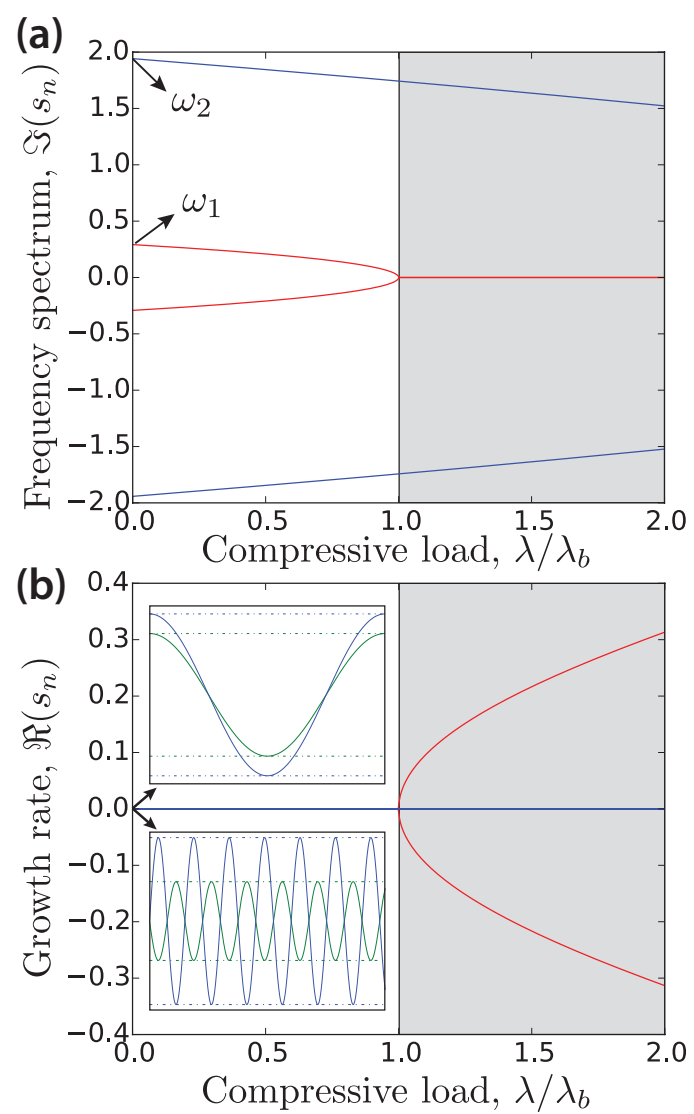

Fig. 4 Evolution of the spectrum of the two Floquet forms (or classic harmonic modes) as a function of loading parameter $\lambda$ for $\eta=0$ and $\beta=0$. (a) Evolution of the natural frequencies of the bi-articulated bar in compression. (b) Evolution of the growth rate of the two modes. The grey regions in (a) and (b) indicate that the trivial state $\theta_{1}^{0}(\tau)=\theta_{2}^{0}(\tau)=0$ is locally unstable. Insets: Top and bottom respectively show, for $\lambda=0$, the angles $\theta_{1}^{*}(\tau)$ and $\theta_{2}^{*}(\tau)$ of the in-phase and outof-phase classic modes with natural frequency $\omega_{1}$ and $\omega_{2}$, respectively. The dashed-dotted lines represent the moduli of the complex eigenfunctions $\left|\mathbf{p}_{n}^{0}\right|$ and $-\left|\mathbf{p}_{n}^{0}\right|$ that envelope the motion.

From Eq.(11) we see that the lines and rows of the truncated Hill matrix of Eq.(10) are independent, whatever the truncation order $H$. If $H=0$, no spectral sorting is needed as the Hill matrix reduces to its central block $\mathbf{J}^{0}$ whose spectrum gives $N=4$ eigenvalues and eigenvectors that correspond to the $N$ classic harmonic modes $\mathbf{y}_{n}(\tau)=\mathbf{p}_{n}^{0} e^{s_{n} \tau}$ when replaced in Eq.(8). If $H>0$, the Hill matrix leads to $(2 H+1)$ identical families of $N$ independent eigenvalues and eigenvectors of $\mathbf{J}^{0}$. Applying the eigenvector sorting method would give the $N$ correct harmonic modes but the eigenvalue sorting algorithm will return an empty spectrum as the computed eigenvalues will never be in the spectral primitive cell $-\beta / 2 \leq \Im\left(s_{l}\right)<\beta / 2$ since $\beta=0$. Thus, only the eigenvector sorting algorithm convey the notion that classic normal modes are particular cases of fundamental FFs. 
Fig. 4 is the classical dynamical vision of buckling. It shows the evolution of the computed spectrum of the $N=4$ harmonic FFs as a function of dimensionless compressive load $\lambda / \lambda_{b}$ where $\lambda_{b}$ is the already mentioned buckling load. At $\lambda=0$, the bi-articulated beam has two classic harmonic vibrational modes in the physical space: one with natural frequency $\omega_{1}$ where the linearized angles $\theta_{1}^{*}(\tau)$ and $\theta_{2}^{*}(\tau)$ vibrates in phase; one with natural frequency $\omega_{2}$ where $\theta_{1}^{*}(\tau)$ and $\theta_{2}^{*}(\tau)$ are out-of-phase. The time evolutions of the linearized angles $\theta_{1}^{*}(\tau)$ and $\theta_{2}^{*}(\tau)$ of those two modes are shown in the insets of Fig. 4(b). The dash-dotted lines represent the constant amplitude of the eigenfunction modulus $\left|\mathbf{p}_{n}^{0}\right|$ whose value is undefined, unless normalized. The free vibratory response of the bi-articulated beam, solution of Eq. (5) with the Jacobian $\mathbf{J}(\tau)$ given in Eq.(11), can be written as a linear superposition of these two normal modes. As the compressive load $\lambda$ is increased, the frequencies of the two fundamental FFs decrease until the smallest one eventually goes to zero at $\lambda=\lambda_{b}$. The lock-in of the conjugate spectrum $s_{1}$ and $\bar{s}_{1}$ on the real axis induces a positive growth rate $\Re\left(s_{1}\right)>0$ and therefore a bifurcation of the trivial spatial straight state of the Ziegler column along the in-phase static mode. This local instability is the one shown in Fig. $3(\mathrm{a})$ and is responsible for the nonlinear response shown in Fig. 2(a). Note that in this case, the two FFs are uncoupled in the physical space as highlighted in Fig. 4 by the fact that the two color lines never combine.

\subsection{Periodic elastic state $(\beta \neq 0)$}

When $\beta \neq 0$, the straight bi-articulated bar is in a periodic elastic state as the Jacobian $\mathbf{J}(\tau)$ of Eq. $(5)$ is $\bar{T}$ periodic with $\bar{T}=2 \pi / \beta$. In the particular conservative case where $\eta=0$, the Hill matrix of Eq.(10), truncated to the order $H=2$, becomes

$\mathbf{H}^{2}=\left[\begin{array}{ccccc}\mathbf{J}^{0}+i 2 \beta \mathbf{1}_{4} & \mathbf{J}^{1} & \mathbf{0}_{4} & \mathbf{0}_{4} & \mathbf{0}_{4} \\ \mathbf{J}^{1} & \mathbf{J}^{0}+i \beta \mathbf{1}_{4} & \mathbf{J}^{1} & \mathbf{0}_{4} & \mathbf{0}_{4} \\ \mathbf{0}_{4} & \mathbf{J}^{1} & \mathbf{J}^{0} & \mathbf{J}^{1} & \mathbf{0}_{4} \\ \mathbf{0}_{4} & \mathbf{0}_{4} & \mathbf{J}^{1} & \mathbf{J}^{0}-i \beta \mathbf{1}_{4} & \mathbf{J}^{1} \\ \mathbf{0}_{4} & \mathbf{0}_{4} & \mathbf{0}_{4} & \mathbf{J}^{1} & \mathbf{J}^{0}-i 2 \beta \mathbf{1}_{4}\end{array}\right]$

with

$\mathbf{J}^{0}=\left[\begin{array}{cccc}0 & 0 & 1 & 0 \\ 0 & 0 & 0 & 1 \\ -\frac{3}{2} & \frac{15}{14} & 0 & 0 \\ 3 & -\frac{33}{14} & 0 & 0\end{array}\right]$ and $\mathbf{J}^{1}=\lambda\left[\begin{array}{cccc}0 & 0 & 0 & 0 \\ 0 & 0 & 0 & 0 \\ \frac{8}{7} & -\frac{12}{7} & 0 & 0 \\ -\frac{12}{7} & \frac{32}{7} & 0 & 0\end{array}\right]$.

Unlike in Eq.(11), the lines and rows of the truncated $\mathbf{H}^{H}$ of Eq.(12) are now fully coupled via the harmonic
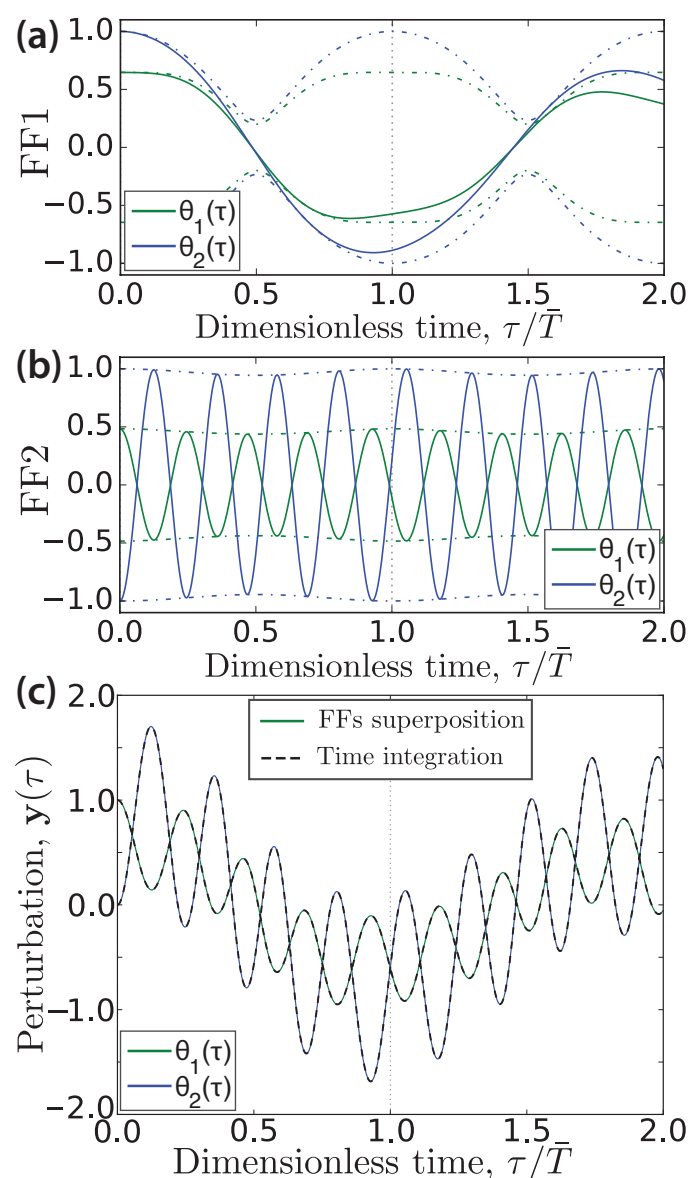

Fig. 5 Vibratory response for $\eta=0, \beta=1.55 \omega_{1}, \lambda=0.75 \lambda_{b}$ and $H=3$. (a) Time evolution of the angles $\theta_{1}^{*}(\tau)$ and $\theta_{2}^{*}(\tau)$ of the first fundamental Floquet form $\mathbf{y}_{1}(\tau)=\mathbf{p}_{1}(\tau) e^{s_{1} \tau}$ over the first two periods $2 \bar{T}$ where $\bar{T}=2 \pi / \beta$. The dash-dotted lines show the moduli of the periodic eigenfunctions $\left|\mathbf{p}_{n}(\tau)\right|$ and $-\left|\mathbf{p}_{n}(\tau)\right|$ that envelope the almost periodic motions. (b) Same as (a) but for the second fundamental Floquet form. (c) Time evolution of the angles $\theta_{1}^{*}(\tau)$ and $\theta_{2}^{*}(\tau)$ of the free vibratory response $\mathbf{y}(\tau)$ of Eq. (5) for the initial conditions $\theta_{1}^{*}(0)=1$ and $\theta_{2}^{*}(0)=\dot{\theta}_{1}^{*}(0)=\dot{\theta}_{2}^{*}(0)=0$. The response has been computed either with a classic direct iterative ODE solver (dashed line) or by recombining the FFs (full line).

contribution of the Jacobian $\mathbf{J}^{1}$ and the sorting of the spectrum of Hill's matrix given in Eq.(12) is a necessity to compute the $N=4 \mathrm{FFs}$ in the state space.

Figs. 5(a),(b) show the two fundamental FFs, $\mathbf{y}_{n}(\tau)=$ $\mathbf{p}_{n}(\tau) e^{s_{n} \tau}$, computed with the eigenvector sorting algorithm, about the trivial spatial state $\theta_{1}^{0}(\tau)=\theta_{2}^{0}(\tau)=0$ for $\eta=0, \beta=1.55 \omega_{1}, \lambda=0.75 \lambda_{b}$ and $H=3$. Those two typical physical FFs are the periodically modulated generalization of the classic harmonic modes shown in the insets of Fig. 4(b). Notably, the first FF of Fig. 5(a) almost-periodically vibrates with a $\theta_{1}^{*}(\tau)$ and $\theta_{2}^{*}(\tau)$ in phase and a fundamental frequency close to $\omega_{1}$ when the second FF in Fig. 5(b) vibrates out-of-phase with a fundamental frequency close to $\omega_{2}$. Unlike classic har- 

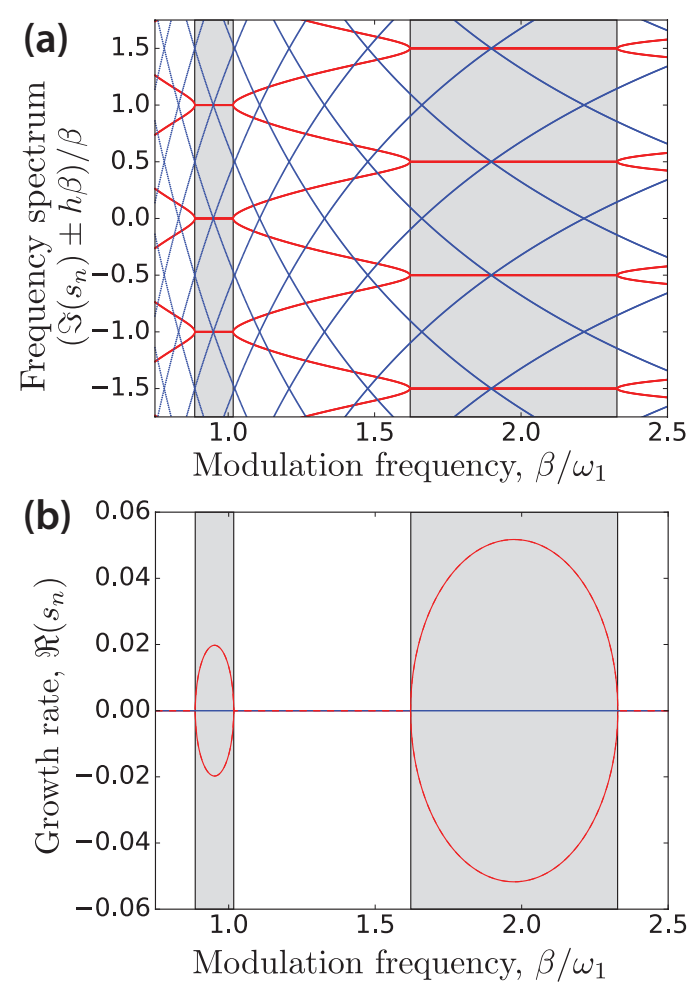

Fig. 6 Evolution of the spectrum of the $N=4$ fundamental FFs as a function of $\beta / \omega_{1}$ for $\eta=0, \lambda=0.75 \lambda_{b}$ and $H=$ 25. (a) Evolution of the frequency spectrum location of the FFs, $\Im\left(s_{n}\right)+\sum_{h} i h \beta$. (b) Evolution of the growth rate of the FFs, $\Re\left(s_{n}\right)$. The grey regions in (a) and (b) indicate that the straight bi-articulated bar is unstable.

monic modes, the modulus of the eigenfunction $\mathbf{p}(\tau)$, whose value is undefined unless normalized, is not constant but $\bar{T}$-periodic as illustrated by the dashed-dotted lines in Figs. 5(a),(b). Like for $\beta=0$, the moduli $|\mathbf{p}(\tau)|$ and $-|\mathbf{p}(\tau)|$ envelope the almost-periodic motion. The superposition property of FFs given in Eq.(6) is highlighted in Fig. 5(c) where we show the free vibratory response of the straight bi-articulated bar computed either through FFs or classic ODE time integrator. The perturbation $\mathbf{y}(\tau)$, solution of Eq.(5), can be decomposed in the normal basis of its FFs which notably means it would have been possible to find a set of initial conditions for which only one FF contributes to the solution $\mathbf{y}(\tau)$ (e.g. in Fig. 5 for $\theta_{1}^{*}(0)=0.646, \theta_{2}^{*}(0)=1$ and $\dot{\theta}_{1}^{*}(0)=\dot{\theta}_{2}^{*}(0)=0$, only the first FF would contribute to the linear response and Fig. 5(c) would have been identical to Fig. 5(a)).

Fig. 6 shows the evolution of the $N=4$ spectra $s_{n}+\sum_{h} i h \beta$ of the computed fundamental FFs as a function of $\beta / \omega_{1}$ for $\lambda=0.75 \lambda_{b}$ and $H=25$ (to ensure convergence). Like in the classic case illustrated in Fig. 4 for $\beta=0$, the fundamental frequencies $\Im\left(s_{n}\right)$, and moreover the frequency spectra $\Im\left(s_{n}\right)+\sum_{h} i h \beta$, vary with the modulation parameters of the elastic state $\lambda$ and $\beta$. Also, since the system is conservative, the two FFs are uncoupled in the physical space as highlighted in Fig. 6 by the independence between the red and blue modal colors. Furthermore, like the classic harmonic modes of Fig. 4, it exists some modulation parameters for which the straight bi-articulated bar becomes unstable, i.e. the spectrum of the in-phase FF $\Im\left(s_{n}\right)+\sum_{h} i h \beta$ lock in the state space with its conjugate leading to a positive associated growth rate $\Re\left(s_{n}\right)$ (we do not see here the lock-in of the out-of-phase FF in Fig. 6 because the latter happens at higher modulation frequency than the displayed one). As illustrated in Figs. 3(c),(d) and displayed in Fig. 6(a), FFs have a poly-harmonic spectrum $\Im\left(s_{n}\right)+\sum_{h} i h \beta$ and can lock either in $i(\beta \pm m \beta)$ or in $i(\beta / 2 \pm m \beta)$. These two phenomena respectively correspond to a $\bar{T}$ and $2 \bar{T}$-periodic FF that diverges exponentially and is responsible for the steady and flip bifurcation shown for example in Fig. 2(b). The principal region of instability, i.e. the one with the largest
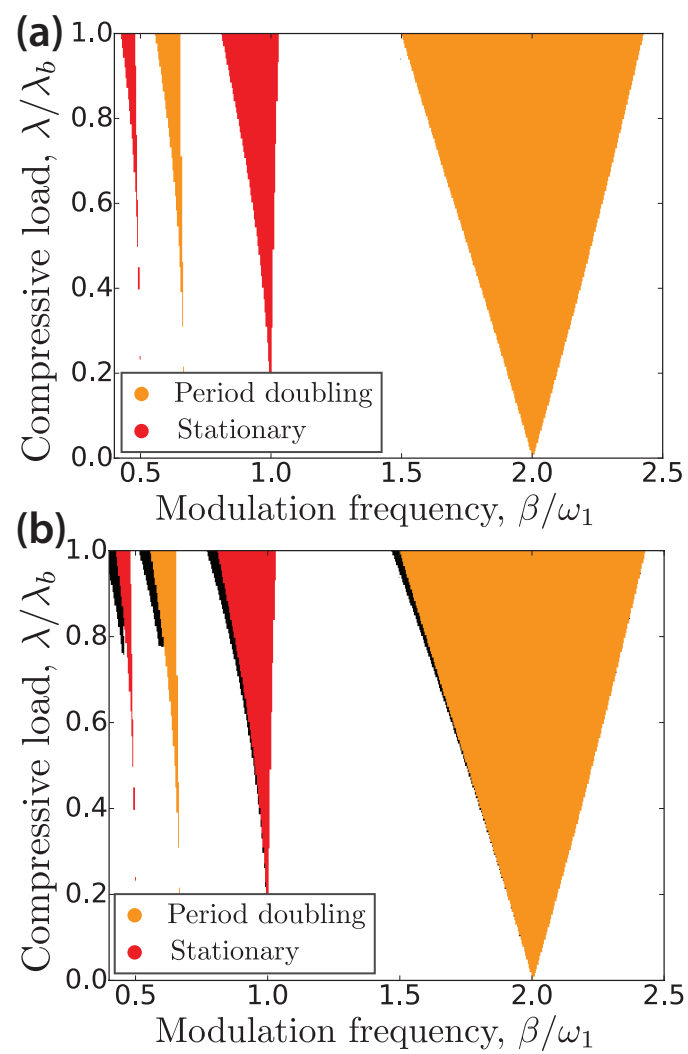

Fig. 7 Stability chart of the conservative system in the $(\lambda, \beta)$ space for $\eta=0, \beta \neq 0$ and a variable converged truncation order $H$ given in Fig. 14(a). (a) $T$ (red) and $2 T$-instability (orange) regions computed through the eigenvector or eigenvalue sorting algorithms. (b) Same stability chart but by analyzing all the spectrum of the Hill matrix, i.e. with no sorting of the eigenvectors or eigenvalues. The black color show supplementary instability regions which are spurious numerical results due to the truncation of the Hill matrix that would happen whatever $H$. 
$\beta$ range, corresponds to a lock-in of the fundamental frequency of a FF when secondary regions are related to lock-in of subharmonics.

Fig. 7(a) displays the stability chart of the straight bi-articulated bar with an end compressive horizontal force in the modulation parameters space $(\lambda, \beta)$. For each parameters, we chose a truncation order $H$ so that the maximum difference $\delta$ between the $N=4$ Floquet multipliers computed with the eigenvector sorting method and the ones obtained from a converged monodromy matrix algorithm [30] is $\delta=1 \times 10^{-6}$. The resulting minimal truncation order map, $H$, as a function of $(\lambda, \beta)$ to ensure convergence is displayed in Fig. 14(a) of Appendix 4. Colored regions, or Math-
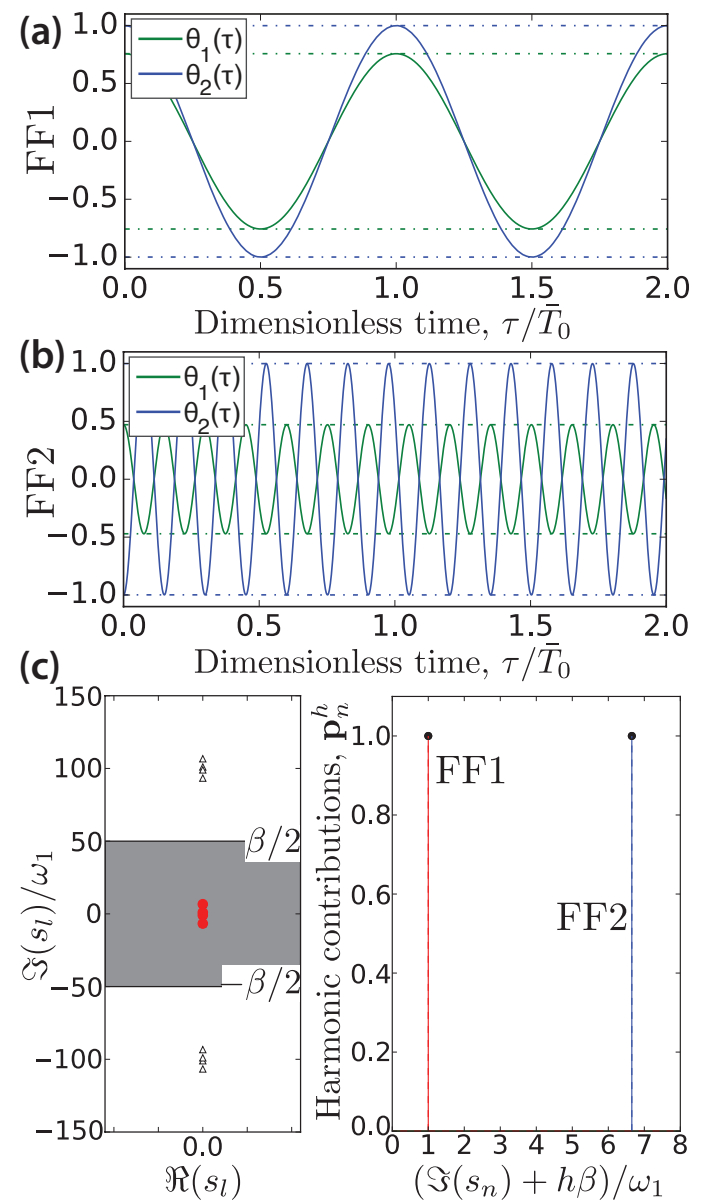

Fig. 8 Fundamental FFs for $\eta=0, \beta=100 \omega_{1}, \lambda=0.75 \lambda_{b}$ and $H=1$. (a) Time evolution of the angles $\theta_{1}^{*}(\tau)$ and $\theta_{2}^{*}(\tau)$ of FF1 over the first two natural periods $2 \bar{T}_{0}$ where $\bar{T}_{0}=2 \pi / \omega_{1}$. The dash-dotted lines show the moduli of the periodic eigenfunctions $\left|\mathbf{p}_{n}(\tau)\right|$ and $-\left|\mathbf{p}_{n}(\tau)\right|$ that envelope the almost periodic motion. (b) Same as (a) but for FF2. (c) (left) Raw spectrum of the truncated Hill matrix. The $N=4$ fundamental Floquet exponents computed by eigenvector sorting are shown with red circles. The region $-\beta / 2 \leq \Im\left(s_{l}\right)<\beta / 2$ for eigenvalue sorting is shown in grey. (right) FFT of the two quasi-harmonic FFs. ieu tongues, that theoretically originate at $2 \beta / m \omega_{1}$ for $\lambda \rightarrow 0$ with $m$ a positive integer, correspond to parameters for which at least one of the growth rate $\Re\left(s_{n}\right)$ of the 4 FFs is positive. Red regions are $T$-instability domains associated with lock of the frequency spectrum of the in-phase $\mathrm{FF}$ in $\beta \pm m \beta$ when orange regions show $2 T$-instability zones related to a $\beta / 2 \pm m \beta$ lock-in of the in-phase FF as shown in Fig. 6. In the particular conservative case $\eta=0$ and for the same truncation order map of $H$ given in Fig. 14(a), the use of the eigenvalue sorting algorithm lead to the exact same stability chart displayed in Fig. 7(a). Indeed, we observe the eigenvector sorting method gives the $N=4$ Floquet exponents $s_{n}$ that are in the primitive spectral cell $-\beta / 2 \leq s_{n}<\beta / 2$ when in $\bar{T}$ or $2 \bar{T}$-periodic instability regions. Fig. 7 (b) shows the stability chart but by using no sorting algorithms, i.e. by analyzing all the eigenvalues $s_{l}$ of the Hill matrix to see whether $\Re\left(s_{l}\right)>0$. The differences between both stability charts are highlighted in black. Whatever the truncation order $H$, the truncated Hill matrix will always give some spurious eigenvalues that are inherent to the harmonic balance method. Those spurious eigenvalues are more visible for high $\lambda$ and close to the instability regions.

\subsection{Asymptotic cases $(\beta \rightarrow+\infty)$ and $(\beta \rightarrow 0)$}

In the asymptotic cases where $\beta \rightarrow+\infty$ or $\beta \rightarrow 0$, i.e. in the situations where the modulation time scale is far from the natural time scale of the system given here by $\omega_{1}$ and $\omega_{2}$, several qualitative and quantitative comments can be made about the FFs.

Fig. 8 illustrates the $\beta \rightarrow+\infty$ scenario by showing the two fundamental FFs of the straight bi-articulated bar in periodic elastic state for $\eta=0, \lambda=0.75 \lambda_{b}$ and $\beta=100 \omega_{1}$. The time evolution of the two angles $\theta_{1}^{*}(\tau)$ and $\theta_{2}^{*}(\tau)$ of the two FFs are shown in Figs. 8(a) and (b). For high modulation frequencies, the harmonic contribution of the compressive force is averaged out and the bi-articulated elastic bar behaves like a classic effective oscillator. The two FFs asymptotically tend to the two classic in-phase and out-of-phase modes of the system with natural frequencies $\omega_{1}$ and $\omega_{2}$ as shown in the insets of Fig. 4(b). Notably, the $\bar{T}$-periodic envelopes of the almost-periodic FFs, $|\mathbf{p}(\tau)|$ and $-|\mathbf{p}(\tau)|$, appear constant over the natural period $\bar{T}_{0}$ since the small oscillations over $\bar{T}$ are negligible. Fig. 8(c) displays the raw spectrum of the Hill matrix (left) as well as the reconstructed spectrum of the FFs (right). In the $\beta \rightarrow+\infty$ case, the FFs tend to classic harmonic modes with a spectrum composed of a single oscillation frequency. This spectrum is easily recovered with 

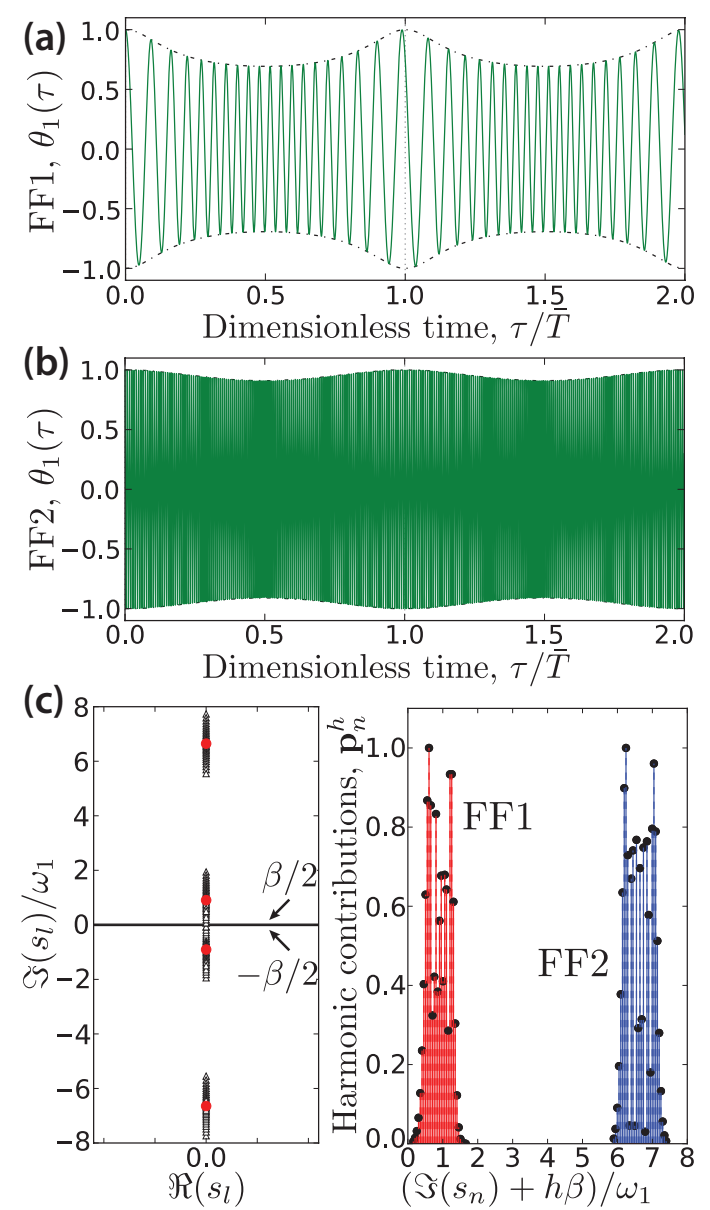

Fig. 9 Fundamental FFs for $\eta=0, \beta=0.05 \omega_{1}, \lambda=0.75 \lambda_{b}$ and $H=15$. (a) Time evolution of the angle $\theta_{1}^{*}(\tau)$ of FF1 over the first two periods $2 \bar{T}$ where $\bar{T}=2 \pi / \beta$ with their envelopes $\left|\mathbf{p}_{n}(\tau)\right|$ and $-\left|\mathbf{p}_{n}(\tau)\right|$. (b) Same as (a) but for FF2. (c) (left) Raw spectrum of the truncated Hill matrix. The $N=4$ fundamental Floquet exponents computed by eigenvector sorting are shown with red circles. The region $-\beta / 2 \leq \Im\left(s_{l}\right)<\beta / 2$ for eigenvalue sorting is shown in grey. (right) FFT of the two FFs.

both eigenvector and eigenvalue sorting algorithms. Actually, both methods lead to the same eigenvalue output as the $N=4$ fundamental Floquet exponents obtained with the eigenvector sorting algorithm and represented by red circle in Fig. 8(c), are the one inside the $-\beta / 2 \leq \Im\left(s_{l}\right)<\beta / 2$.

Fig. 9 illustrates the $\beta \rightarrow 0$ situation by showing the two in-phase and out-of-phase FFs of Fig. 8, but for a very small modulation frequency $\beta=0.05 \omega_{1}$. The time evolution of the angle $\theta_{1}^{*}(\tau)$ of the two FFs are shown in Figs. $9(\mathrm{a})$ and $(\mathrm{b})\left(\theta_{2}^{*}(\tau)\right.$ is not displayed for a sake of clarity). For slow modulation frequency, the end compressive load quasi-statically takes all the amplitudes between the minimum $\lambda=-0.75 \lambda_{b}$ and maximum $+0.75 \lambda_{b}$. As a consequence, the elasticity of our system is quasi-statically modulated and the trans- verse natural frequencies of the straight bi-articulated bar appear to almost continuously vary (with a small step $\beta$ ) around $\omega_{1}$ and $\omega_{2}$. We can see on Figs. 9(a) and (b) that the motion is modulated in frequency and amplitude. The FFTs of the two FFs are displayed on the right side of Fig. 9(c) and show two broad spectrum in the vicinity of $\omega_{1}$ and $\omega_{2}$ whose almost continuous width depend on the modulation amplitude $\lambda$. For small $\beta$, many frequencies are contained in the FFs and a high truncation order $H$ is needed for the spectrum of Hill's matrix to converge. The raw spectrum of Hill matrix is shown on the left of Fig. 9(c) for $H=15$. It is separated in four distinct packets, centered around $\omega_{1}, \omega_{2},-\omega_{1}$ and $-\omega_{2}$, that correspond to $N=4$ families of eigenvalues $s_{n+k}=s_{n}+i k \beta$ where $-H \leq k \leq+H$ (apart from the edges of the packets where the computed eigenvalues are spurious due to truncation errors). The eigenvector sorting algorithm finds the $N=4$ fundamental Floquet exponents, highlighted by red circles, that correspond to the most converged eigenvalues (center of the packets). Because of the small modulation frequency $\beta$, the eigenvalue sorting algorithm is far from being converged for $H=15$ as only two, yet not converged, eigenvalues relative to the first FFs are located in the $-\beta / 2 \leq \Im\left(s_{l}\right)<\beta / 2$ region. For the two eigenvalues associated with the spectrum packet around $\omega_{2}$ and $-\omega_{2}$ to enter that region, more than $H=100$ would be needed. In the limit situation $\beta \rightarrow 0$, the eigenvalue sorting algorithm is not adapted to efficiently compute the FFs and their spectrum, on the contrary to the eigenvector sorting method that computes the most converged fundamental Floquet exponents and FFs whatever $\beta$. In the non-conservative case studied in next section, the same kind of convergence issue will happen in the determination of instability regions associated with Neimark-Sacker bifurcation when using the eigenvalue sorting algorithm.

\section{Non-Conservative case $(\eta=1)$}

In this section, we analyze the influence of the modulation loading parameters $\beta$ and $\lambda$ on the transverse vibrational modes of the Ziegler column under a nonconservative end positional loading $(\eta=1)$. We start with the classic case of a constant compressive force, i.e. for $\beta=0$, and study the influence of periodicity when $\beta \neq 0$ on the modes and stability.

\subsection{Constant elastic state $(\beta=0)$}

In the particular case $\beta=0$, the applied compressive dimensionless load $\bar{P}(\tau)=\lambda \cos (\beta \tau)$ and therefore the 
periodically time-varying Jacobian $\mathbf{J}(\tau)$ of Eq.(5) are independent of time. The Hill matrix of Eq.(10) truncated to the order $H$ reduces to a block diagonal matrix with only the fundamental part of the Jacobian $\mathbf{J}^{0}$ as shown in Eq.(11). In this section, since $\eta=1$, the Jacobian $\mathbf{J}^{0}$ reads,

$\mathbf{J}^{0}=\left[\begin{array}{cccc}0 & 0 & 1 & 0 \\ 0 & 0 & 0 & 1 \\ -\frac{3}{2} & \frac{15}{14} & 0 & 0 \\ 3 & -\frac{33}{14} & 0 & 0\end{array}\right]+\lambda\left[\begin{array}{cccc}0 & 0 & 0 & 0 \\ 0 & 0 & 0 & 0 \\ \frac{8}{7} & -\frac{5}{7} & 0 & 0 \\ -\frac{12}{7} & \frac{12}{7} & 0 & 0\end{array}\right]$.

We can infer from the shape of the Jacobian in Eq.(13) that the problem is now non-conservative as the bottom left $2 \times 2$ block matrix of $\mathbf{J}^{0}$ depending on $\lambda$ is non symmetric. Whether we compute the whole spectrum of $\mathbf{J}^{0}$, i.e. the truncated Hill matrix for $H=0$, or we use the eigenvector sorting algorithm for $H>0$ (like in the conservative case, the eigenvalue sorting algorithm can not be used for $\beta=0$ ), one numerically approximates $N=4$ FFs for a given modulation parameter $\lambda$.
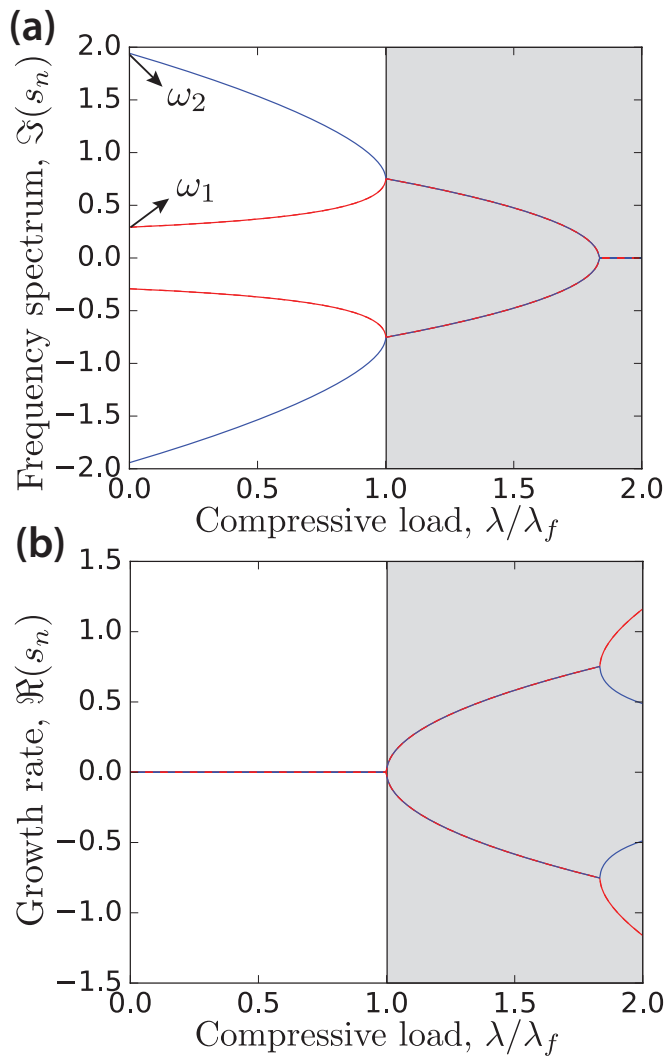

Fig. 10 Evolution of the spectrum of the two Floquet forms (or classic harmonic modes) as a function of loading parameter $\lambda$ for $\eta=1$ and $\beta=0$. (a) Evolution of the natural frequencies of the bi-articulated bar in compression. (b) Evolution of the growth rate of the two modes. The grey regions in (a) and (b) indicate that the trivial state $\theta_{1}^{0}(\tau)=\theta_{2}^{0}(\tau)=0$ is locally unstable.
Fig. 10 shows the plot of the evolution of the computed spectrum of the $N=4$ fundamental FFs as a function of dimensionless compressive load $\lambda / \lambda_{f}$ where $\lambda_{f}$ is the already mentioned flutter load. At $\lambda=0$, the unloaded straight bi-articulated bar has two classic inphase and out-of-phase vibrational modes with natural frequency $\omega_{1}$ and $\omega_{2}$, respectively. Unlike the conservative case, the two harmonic FFs are coupled for $\eta=1$ and influence each other so that their spectra eventually lock-in. As the compressive load $\lambda$ grows, the frequency of the in-phase mode increases when the one of the outof-phase mode decreases. At $\lambda=\lambda_{f}$, the spectra of the two physical FFs lock in a finite $\Im\left(s_{n}\right)>0$, inducing a positive growth rate $\Re\left(s_{n}\right)$ of the resulting locked mode
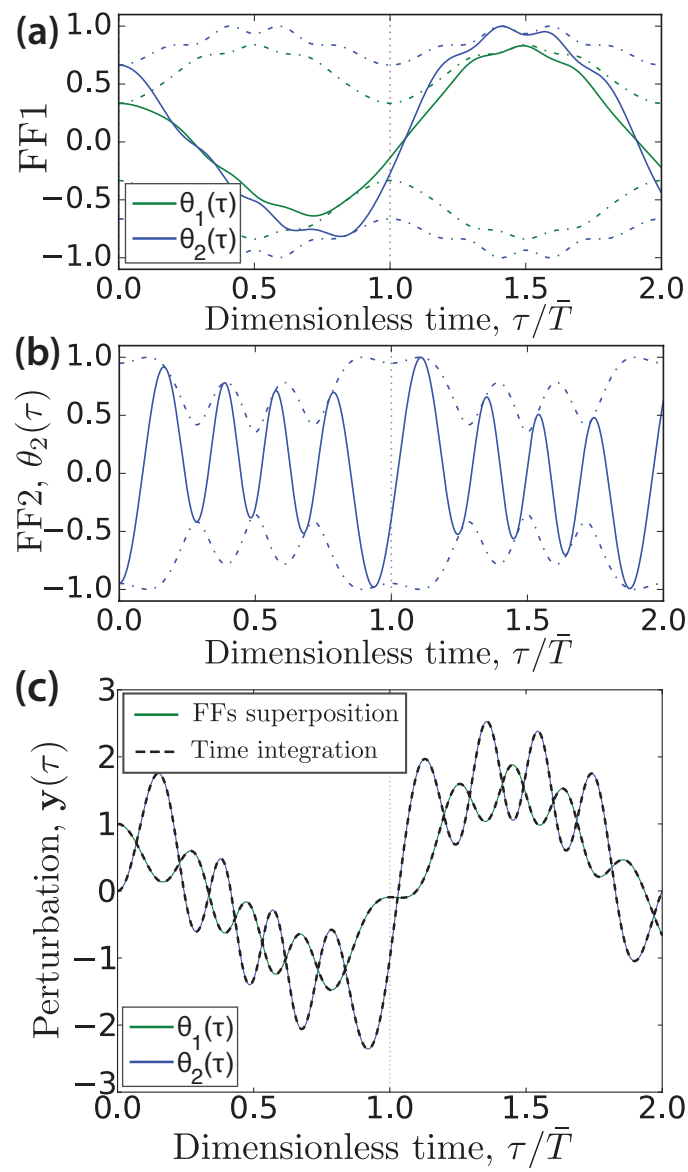

Fig. 11 Vibratory response for $\eta=1, \beta=1.55 \omega_{1}, \lambda=$ $0.75 \lambda_{f}$ and $H=5$. (a) Time evolution of the angles $\theta_{1}^{*}(\tau)$ and $\theta_{2}^{*}(\tau)$ of the first fundamental FF $\mathbf{y}_{1}(\tau)=\mathbf{p}_{1}(\tau) e^{s_{1} \tau}$ over the first two periods $2 \bar{T}$ where $\bar{T}=2 \pi / \beta$. The dash-dotted lines show the moduli of the periodic eigenfunctions $\left|\mathbf{p}_{n}(\tau)\right|$ and $-\left|\mathbf{p}_{n}(\tau)\right|$ that envelope the almost periodic motions. (b) Same as (a) but for the second fundamental FF (only $\theta_{2}^{*}(\tau)$ is shown for a sake of clarity). (c) Time evolution of the angles $\theta_{1}^{*}(\tau)$ and $\theta_{2}^{*}(\tau)$ of the free vibratory response $\mathbf{y}(\tau)$ of Eq. (5) for the initial conditions $\theta_{1}^{*}(0)=1$ and $\theta_{2}^{*}(0)=\dot{\theta}_{1}^{*}(0)=\dot{\theta}_{2}^{*}(0)=0$. The response has been computed either with a classic direct iterative ODE solver or by recombining the FFs. 
(for $\lambda>\lambda_{f}$, only one in-phase mode subsists in the physical space which separates in an unstable and stable one in the state space). This destabilization mechanism corresponds to the flutter instability case shown in Fig. 3(b) that is responsible for the Hopf bifurcation illustrated in Fig. 2(c). Increasing $\lambda$ further, the spectra of the remaining FF lock again, this time in the state space and on $\Im\left(s_{n}\right)=0$. This second situation is qualitatively similar to the instability by divergence shown in Fig. 4 and Fig. 3(a) for the conservative case. The flutter instability only happens on a range of loading parameter $\lambda$ and evolves toward a divergence instability for sufficiently high compressive loads.

\subsection{Periodic elastic state $(\beta \neq 0)$}

When $\beta \neq 0$, the straight Ziegler column is in a periodic elastic state and its perturbed motion is governed by Eq.(5) with a $\bar{T}$-periodic Jacobian $\mathbf{J}(\tau)$ where $\bar{T}=2 \pi / \beta$. The truncated matrix has therefore the shape of a tridiagonal matrix by block as shown in Eq.(12) but since $\eta=1$ in this section, the fundamental and first harmonic contributions of $\mathbf{J}(\tau)$ now reads

$\mathbf{J}^{0}=\left[\begin{array}{cccc}0 & 0 & 1 & 0 \\ 0 & 0 & 0 & 1 \\ -\frac{3}{2} & \frac{15}{14} & 0 & 0 \\ 3 & -\frac{33}{14} & 0 & 0\end{array}\right]$ and $\mathbf{J}^{1}=\lambda\left[\begin{array}{cccc}0 & 0 & 0 & 0 \\ 0 & 0 & 0 & 0 \\ \frac{8}{7} & -\frac{5}{7} & 0 & 0 \\ -\frac{12}{7} & \frac{12}{7} & 0 & 0\end{array}\right]$

We use the eigenvector sorting algorithm on the spectrum of the truncated Hill matrix with $\mathbf{J}^{0}$ and $\mathbf{J}^{1}$ given in Eq.(14) to compute the $N=4$ FFs of the straight Ziegler column under a given end following compressive load $\bar{P}(\tau)=\lambda \cos (\beta \tau)$.

Figs. 11(a),(b) display the perturbed angles $\theta_{1}^{*}(\tau)$ and $\theta_{2}^{*}(\tau)$ of two fundamental FFs, in the form $\mathbf{y}_{n}(\tau)=$ $\mathbf{p}_{n}(\tau) e^{s_{n} \tau}$, about the trivial spatial state $\theta_{1}^{0}(\tau)=\theta_{2}^{0}(\tau)=$ 0 for $\eta=1, \beta=1.55 \omega_{1}, \lambda=0.75 \lambda_{f}$ and $H=5$. Like in the conservative case $\eta=0$ illustrated in Fig. 5 , the FFs are the almost-periodic generalization of the classic harmonic modes shown in the insets of Fig. 10(b). For $\beta \neq 0$, the eigenfunction $\mathbf{p}_{n}(\tau)$ are periodic and the moduli $\left|\mathbf{p}_{n}(\tau)\right|$ and $-\left|\mathbf{p}_{n}(\tau)\right|$ envelope the oscillation. In the non conservative case with $\eta=1$, the following end compressive force modulates both FFs as illustrated in Fig. 11(a),(b) where the amplitude modulation of the periodic $\left|\mathbf{p}_{1}(\tau)\right|$ and $\left|\mathbf{p}_{2}(\tau)\right|$ are of similar intensity. The superposition property of the normal forms still holds in the non conservative case and is highlighted in Fig. $11(\mathrm{c})$. For a given set of initial condition, the perturbation $\mathbf{y}(\tau)$ solution of Eq.(5) computed with a classic
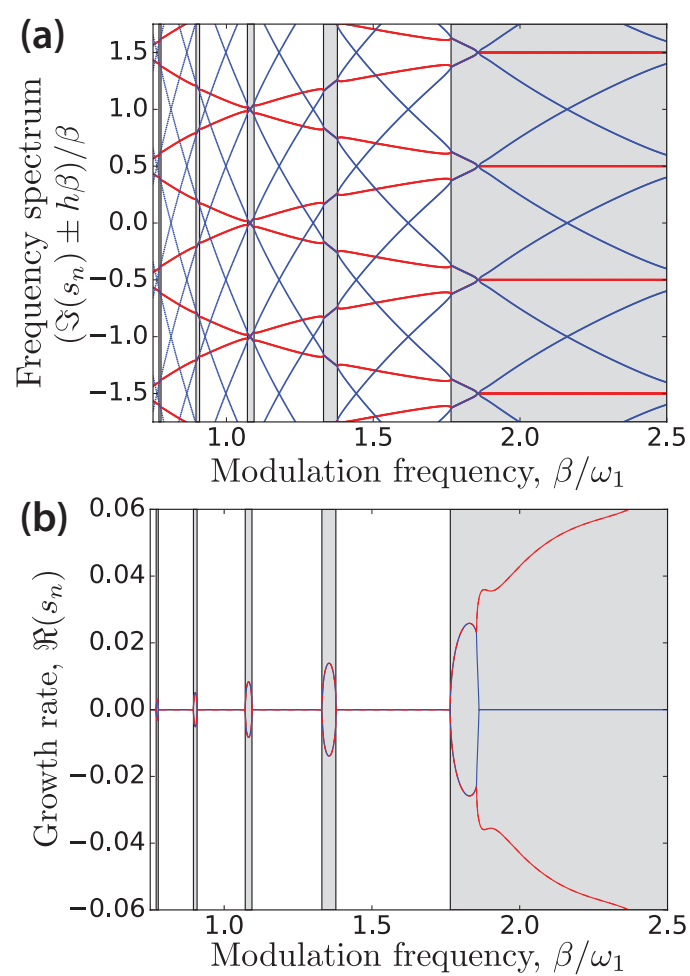

Fig. 12 Evolution of the spectrum of the $N=4$ fundamental FFs as a function of $\beta / \omega_{1}$ for $\eta=1$ and $\lambda=0.75 \lambda_{f}$. (a) Evolution of the frequency spectrum location of the FFs, $\Im\left(s_{n}\right)+\sum_{h} i h \beta$. (b) Evolution of the growth rate of the FFs, $\Re\left(s_{n}\right)$. The grey regions in (a) and (b) indicate that the straight Ziegler column is locally unstable.

direct time ODE solver is in perfect agreement with the solution recombined from FFs with Eq.(6).

Fig. 12 shows the evolution of the $N=4$ spectra $s_{n}+\sum_{h} i h \beta$ of the computed FFs as a function of $\beta / \omega_{1}$ for $\lambda=0.75 \lambda_{f}$ and $H=25$. Similarly to Figs. 4, 6 and 10 , the frequency spectra $\Im\left(s_{n}\right)+\sum_{h} i h \beta$ varies with the modulation parameters $\lambda$ and $\beta$ and can eventually lock-in. Because the FFs have a poly-harmonic spectrum and $\eta=1$, it exists several ranges of frequency modulation $\beta$ where the spectra of two different FFs are locked in frequencies that are not positive integer multiple of $\beta / 2$ (those are the regions where the red and blue colors in Fig. 12 mix and where every growth rates $\Re\left(s_{n}\right)$ are different from zero in Fig. 12(b)). Those situations are qualitatively illustrated in Fig. 3(e) and are responsible for the Neimark-Sacker bifurcation illustrated in Fig. 2(d). The wider region of instability responsible for those secondary-hopf bifurcations corresponds to a lock-in of the fundamental harmonic of the two different FFs when the other ones are due to lockin of sub-harmonics. It is interesting to note that in the main instability region (far right of Fig. 12), the spectra of the two FFs ultimately lock in $i(\beta / 2 \pm m \beta)$ when 

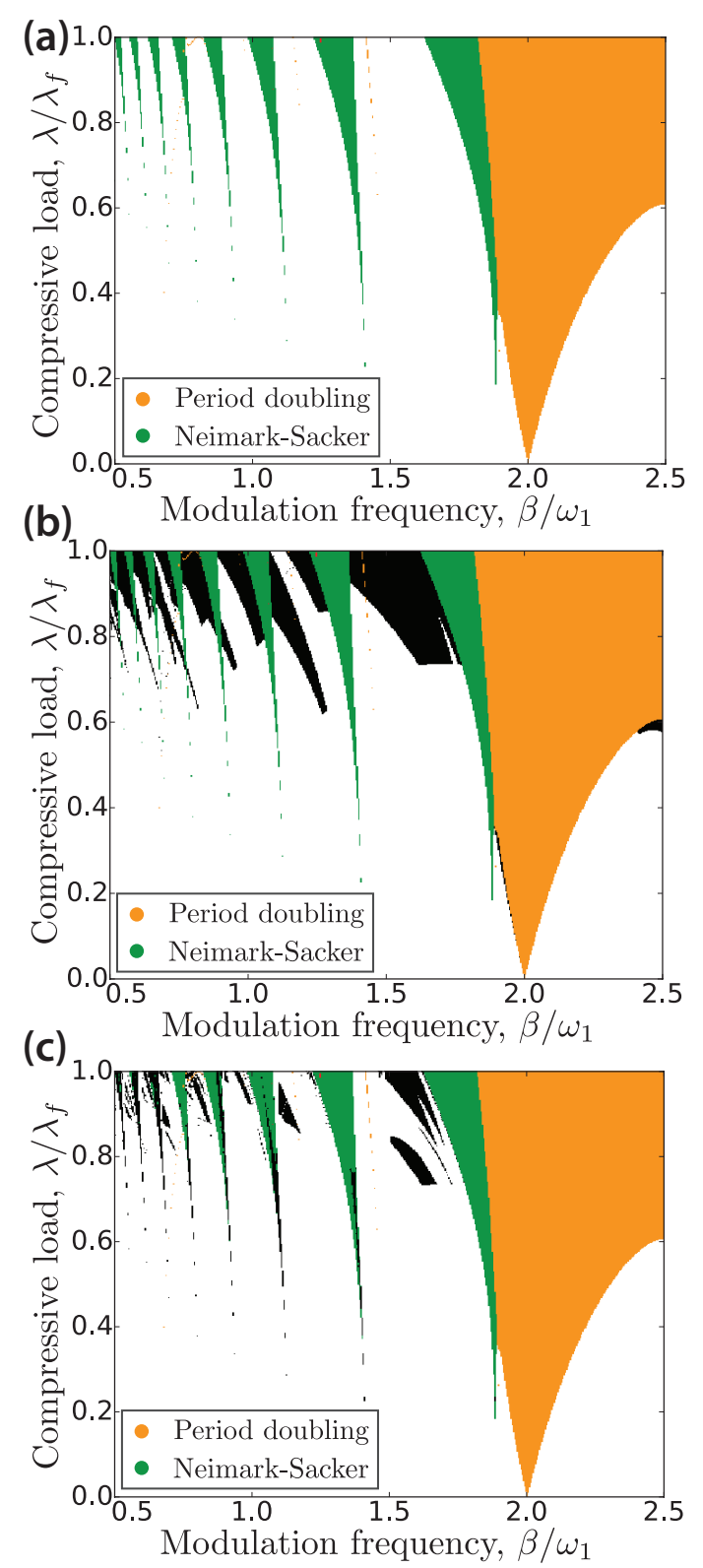

Fig. 13 Stability chart of the non conservative system in the $(\lambda, \beta)$ space for $\eta=1, \beta \neq 0$ and a variable converged truncation order $H$ given in Fig. 14(b). (a) Neimark-Sacker instability (green) and $2 T$-instability (orange) regions computed through the eigenvector sorting algorithm. (b) Same stability chart but by analyzing the raw spectrum of the Hill matrix. The black color shows supplementary instability regions, a spurious numerical artifact inherent to the truncated Hill matrix. (c) Same stability chart but by using the eigenvalue sorting algorithm. The black color indicates supplementary instability regions where the eigenvalue sorting algorithm is not yet converged for the truncation order of Fig. 14(b).

increasing $\beta$, i.e. the system undergoes a $2 \bar{T}$-instability. This main instability was already observed in Fig. 6 .

Fig. 13(a) displays the stability chart of the straight Ziegler column with an end compressive positional following force in the modulation parameters space $(\lambda, \beta)$.
Like in Fig. 7, the displayed stability chart obtained with the eigenvector sorting algorithm has been validated trough the computation of the monodromy matrix and its Floquet multipliers in the time domain [30], leading to a map of truncation order $H$ in the $(\lambda, \beta)$ space shown in Fig. 14(b) of Appendix 4. The system exhibits instability tongues that correspond to parameters for which at least one of the growth rate $\Re\left(s_{n}\right)$ of the $N=4$ fundamental FFs is positive. Here, green regions are associated with secondary Hopf bifurcations and correspond to modulation ranges where the two physical FFs are locked in frequencies that are not integer multiple of $\beta / 2$ when the orange region shows the $2 T$-instability situation related to a $\beta / 2 \pm m \beta$ lock-in of the fundamental of the resulting in-phase FF in the state space as illustrated in Fig. 12. Like in the conservative case in Fig. 7, the analysis of the full spectrum of Hill's matrix (no sorting) leads to a wrong stability diagram displayed in Fig. 13(b). Moreover, unlike for $\bar{T}$ and $2 \bar{T}$-instability regions, the eigenvalue sorting algorithm, represented by the stability chart of Fig. $13(\mathrm{c})$, is not giving the correct Neimark-Sacker instability regions for the optimal truncation order of Fig. 14(b). The reason is that for some modulation parameters and at this optimal truncation order, the sorted Floquet exponents $s_{n}$ that are in the primitive spectral cell $-\beta / 2 \leq s_{n}<\beta / 2$ are not yet converged because they are not the fundamental ones responsible for the Neimark-Sacker lock-in instability. On the contrary, the FFs computed with the eigenvector sorting algorithm are the fundamental ones, a property that is crucial when dealing with secondary Hopf bifurcations or stable FFs for small $\beta$ as explained in Section 3.3.

\section{Conclusions}

The presented study has shown how to practically implement and compute the vibrational modes, or Floquet Forms (FFs), of a structure in periodic elastic state through the archetypal example of a Ziegler column subjected to an end harmonic compressive force. The latter has been used to compare the classic spectral methods that exist to compute the FFs through Hill's matrix. Our results highlighted the absolute necessity to sort the spectrum of Hill's matrix and the benefit of the eigenvector sorting algorithm that selects the fundamental FFs, which are the most converged ones. We also showed the similarities and differences between the classic harmonic modes about equilibria and the almost-periodic FFs about periodic stationary states. Mathematically and physically, FFs can be seen as a poly-harmonic generalization of harmonic modes. At high frequency modulation, FFs tend to effective 
classic vibrational modes as the modulated state is averaged to its fundamental part. On the contrary, at low frequency modulation, a spectral leakage occurs and FFs contain an almost continuous frequency spectrum, with a finite bandwidth. In the particular case of zero frequency modulation, FFs are equivalent to classic harmonic modes where the natural frequencies is specified by the phase of the periodic elastic state.

The spectral calculation of FFs could be used in many engineering systems, from rotating machineries with geometric imperfections to the transverse vibrations of slender structures with compressive or tensile periodic stresses. For practical purposes in computational mechanics though, further numerical developments would be needed for a computation of the converged spectrum of large Hill matrices in reasonable CPU time. Notably, the current eigenvector sorting algorithm applies on the full raw spectrum, an impractical situation for high dimensional systems. It would therefore be crucial to find a way to include this sorting algorithm at the level of the eigenvectors calculation in order to reduce the spectrum computation to the first few physical fundamental FFs. Another possibility would be to take advantage of the nature of the eigenvalue problem and use domain decomposition methods as well as parallelization techniques to improve computational times. Along the development of the modal analysis of structures in periodic states, FFs should be tested as a reduction basis for linear and nonlinear problems in structural vibration or as preferential directors in predictor-corrector algorithms. Another important application lies in the insight given by FFs on dynamic instabilities as shown numerically in this manuscript. Notably, it could be interesting to use the frequency lock-in of FFs as an experimental instability precursor for structures in period states. An experimental Ziegler column [3] would be a relevant test bench to assess the feasibility of instability detections through the spectral signature of FFs.

\section{Appendix 1: Equation of motion of the Ziegler column}

With the help of Newton's second law applied on the two rotating rigid bars parameterized by $\theta_{1}(t)$ and $\theta_{2}(t)$, it is possible to establish the nonlinear equation of motion of the Ziegler column described in Fig. 1. By equating the quantity of acceleration $\mathcal{A}_{b a r 1}$ and $\mathcal{A}_{\text {bar } 2}$ on one side, to the sum of the external moments $\mathcal{M}_{\text {bar } 1}$ and $\mathcal{M}_{\text {bar } 2}$, on the bar 1 and 2, respectively, on the other side, we obtain a system of two nonlinear Ordinary Dif- ferential Equations:

$$
\begin{aligned}
& \mathcal{A}_{\text {bar } 1}=\frac{d}{d t}\left(\frac{\partial \mathcal{T}}{\partial \dot{\theta}_{1}}\right)-\frac{\partial \mathcal{T}}{\partial \theta_{1}}=\mathcal{M}_{1}^{k}+\mathcal{M}_{1}^{c}+\mathcal{M}_{1}^{P} \\
& \mathcal{A}_{\text {bar } 2}=\frac{d}{d t}\left(\frac{\partial \mathcal{T}}{\partial \dot{\theta}_{2}}\right)-\frac{\partial \mathcal{T}}{\partial \theta_{2}}=\mathcal{M}_{2}^{k}+\mathcal{M}_{2}^{c}+\mathcal{M}_{2}^{P}
\end{aligned}
$$

In Eq. $(15), \mathcal{T}\left(\theta_{1}, \theta_{2}, \dot{\theta}_{1}, \dot{\theta}_{2}\right)$ is the kinetic energy of the system of two rotating rigid bars reading

$\mathcal{T}=\frac{8}{3} m l^{2} \dot{\theta}_{1}^{2}+\frac{2}{3} m l^{2} \dot{\theta}_{2}^{2}+2 m l^{2} \dot{\theta}_{1} \dot{\theta}_{2} \cos \left(\theta_{1}-\theta_{2}\right)$.

The moments $\mathcal{M}_{1}^{k}$ and $\mathcal{M}_{1}^{k}$ represent the restoring elastic moments due to the rotational springs and read, in bar 1 and 2, respectively:

$\mathcal{M}_{1}^{k}=k \theta_{1}+k\left(\theta_{1}-\theta_{2}\right)$ and $\mathcal{M}_{2}^{k}=k\left(\theta_{2}-\theta_{1}\right)$.

The moments $\mathcal{M}_{1}^{P}$ and $\mathcal{M}_{2}^{P}$ are due to the end external harmonic force $F(t)$ and read, in bar 1 and 2, respectively:

$$
\begin{aligned}
& \mathcal{M}_{1}^{P}=2 l A \cos (\Omega t)\left[-\cos \left(\theta_{1}\right) \sin \left(\eta \theta_{2}\right)+\sin \left(\theta_{1}\right) \cos \left(\eta \theta_{2}\right)\right] \\
& \mathcal{M}_{2}^{P}=2 l A \cos (\Omega t)\left[-\cos \left(\theta_{2}\right) \sin \left(\eta \theta_{2}\right)+\sin \left(\theta_{2}\right) \cos \left(\eta \theta_{2}\right)\right]
\end{aligned}
$$

If $\eta=0$ (in the case of a force remaining horizontal upon deformation of the structure), the moments $\mathcal{M}_{1}^{P}$ and $\mathcal{M}_{2}^{P}$ can be derived from the gradient of a potential energy, and the system is said to be conservative, or periodically conservative as the value of the moments are periodically varying with time. If $\eta=1$ (in the case of moments depending on the position of the structure in space), the previous property is not true and the system is non-conservative. Replacing Eqs.(16)-(18) into Eq.(15), one obtains the nonlinear system of equation of motions of the two-degrees-of-freedom as expressed in Eq.(1).

\section{Appendix 2: Derivation of the complex Hill ma- trix}

In this section, we explain how to derive the Hill matrix of the time-periodic linearized equation of motion Eq. (5), $\dot{\mathbf{y}}(\tau)=\mathbf{J}(\tau) \mathbf{y}(\tau)$, in the complex domain. The first step consists in rewriting the $\bar{T}$-periodic Jacobian of size $N=4, \mathbf{J}(\tau)=\mathbf{J}(\tau+2 \pi / \beta)$, in a complex Fourier series. In our particular case of a Ziegler column submitted to a harmonic end compressive load, $\mathbf{J}(\tau)$ can be expanded in the closed-form $\mathbf{J}(\tau)=\mathbf{J}^{0}+\mathbf{J}^{-1} e^{-i \beta \tau}+$ $\mathbf{J}^{1} e^{i \beta \tau}$ where $\mathbf{J}^{0}, \mathbf{J}^{1}$ and $\mathbf{J}^{-1}$ are given in Eq.(5).

According to Floquet theory, Floquet forms (FFs) are the $N=4$ particular fundamental solutions $\mathbf{y}(\tau)$ in the form $\mathbf{y}(\tau)=\mathbf{p}(\tau) e^{s \tau}$ where $\mathbf{p}(\tau)=\mathbf{p}(\tau+\bar{T})$ 
and $s$ is a complex number. Since the eigenfunction $\mathbf{p}(\tau)$ is $\bar{T}$-periodic, the FF can also be expanded in the complex Fourier series $\mathbf{y}(\tau)=\sum_{h=-\infty}^{+\infty} \mathbf{p}^{h} e^{(i h \beta+s) \tau}$ and the associated velocity $\dot{\mathbf{y}}(\tau)$ reads $\dot{\mathbf{y}}(\tau)=\sum_{h=-\infty}^{\infty}(s+$ $i h \beta) \mathbf{p}^{h} e^{(s+i h \beta) \tau}$.

Replacing the expanded expressions of $\mathbf{y}(\tau), \dot{\mathbf{y}}(\tau)$ and $\mathbf{J}(\tau)$ in Eq. $(5)$, one can recast the equation of motion from the time domain to the frequency domain such that

$\mathbf{0}=\mathbf{J}(\tau) \mathbf{y}(\tau)-\dot{\mathbf{y}}(\tau)$

$\mathbf{0}=\sum_{h=-\infty}^{\infty}\left[\mathbf{J}^{0} \mathbf{p}^{h} e^{(s+i h \beta) \tau}+\mathbf{J}^{-1} \mathbf{p}^{h} e^{(s+i(h-1) \beta) \tau}\right.$

$$
\left.+\mathbf{J}^{1} \mathbf{p}^{h} e^{(s+i(h+1) \beta) \tau}-(s+i h \beta) \mathbf{p}^{h} e^{(s+i h \beta) \tau}\right]
$$

From Eq.(19), by taking into account that the $e^{s \tau}$ can be factorized and removed, it is now straightforward to apply the harmonic balance method. The latter is based on the property that in order for the sum of all harmonics $e^{i h \beta \tau}$ for $h=-\infty \ldots+\infty$ to be balanced to zero, the sum of the contributions in front of each harmonics $e^{i h \beta \tau}$ has to be balanced to zero. For practical purposes, this remark leads to several vectorial algebraic equations of dimension $N=4$. For the first five harmonics $-2,-1,0,1$ and 2 , those equations read:

$$
\begin{aligned}
& e^{-2 i \beta \tau}: \mathbf{J}^{-1} \mathbf{p}^{-1}+\left(\mathbf{J}^{0}-(s-2 i \beta)\right) \mathbf{p}^{-2}+\mathbf{J}^{1} \mathbf{p}^{-3}=0 \\
& e^{-i \beta \tau}: \mathbf{J}^{-1} \mathbf{p}^{0}+\left(\mathbf{J}^{0}-(s-i \beta)\right) \mathbf{p}^{-1}+\mathbf{J}^{1} \mathbf{p}^{-2}=0 \\
& e^{0 i \beta \tau}: \mathbf{J}^{-1} \mathbf{p}^{1}+\left(\mathbf{J}^{0}-s\right) \mathbf{p}^{0} \quad+\mathbf{J}^{1} \mathbf{p}^{-1}=0 \\
& e^{i \beta \tau}: \quad \mathbf{J}^{-1} \mathbf{p}^{2}+\left(\mathbf{J}^{0}-(s+i \beta)\right) \mathbf{p}^{1}+\mathbf{J}^{1} \mathbf{p}^{0}=0 \\
& e^{2 i \beta \tau}: \mathbf{J}^{-1} \mathbf{p}^{3}+\left(\mathbf{J}^{0}-(s+2 i \beta)\right) \mathbf{p}^{2}+\mathbf{J}^{1} \mathbf{p}^{1}=0
\end{aligned}
$$

Eq.(20) is an eigenvalue problem, truncated to the order $H=2$, that can be transform in the matrix form of Eq.(10), $\left(\mathbf{H}^{2}-s \mathbf{1}\right) \mathbf{q}^{2}=\mathbf{0}$ where $\mathbf{H}^{2}$ is the Hill matrix truncated to the order $H=2$, given in Eq.(12), $s$ is the complex eigenvalue of $\mathbf{H}^{2}$ and $\mathbf{q}^{2}=$ $\left\{\mathbf{p}^{-2} \mathbf{p}^{-1} \mathbf{p}^{0} \mathbf{p}^{1} \mathbf{p}^{2}\right\}^{T}$ is the associated eigenvector. Note that the construction of the complex Hill matrix for higher truncated order $H$ follows the exact same reasoning shown for $H=2$. The problem we encounter when the eigenvalue problem Eq.(20) is truncated, e.g. to the order $H=2$ in the form of the Hill matrix $\mathbf{H}^{2}$, is that we have to drop the contributions $\mathbf{p}^{-3}$ and $\mathbf{p}^{3}$. The consequence is that Eq.(20) are only approximated because some equations are not correct anymore. This is the reason why a sorting algorithm is necessary to compute the Floquet forms of the Hill matrix.

In the general case of a more complex periodic Jacobian than the harmonic one we dealt with in this article, the latter cannot be expanded in a closed-form Fourier series but rather in the general form of Eq.(9),
$\mathbf{J}(\tau)=\sum_{k=-\infty}^{+\infty} \mathbf{J}^{k} e^{i k \beta \tau}$. In this scenario, the aforementioned spectral expansions of $\mathbf{y}(\tau)$ and $\dot{\mathbf{y}}(\tau)$ remain the same but the product $\mathbf{J}(\tau) \mathbf{y}(\tau)$ changes, so that Eq. (19) now becomes

$\mathbf{0}=\sum_{h=-\infty}^{+\infty} \sum_{k=-\infty}^{\infty}\left(\mathbf{J}^{k} \mathbf{p}^{h-k}-(s+i h \beta) \mathbf{p}^{h}\right) e^{i h \beta \tau}$.

Eq.(21) is an infinite value problem that can be recast in the matrix form $\left(\mathbf{H}^{\infty}-s \mathbf{1}\right) \mathbf{q}^{\infty}=\mathbf{0}$ where $\mathbf{H}^{\infty}$ is the general complex infinite Hill matrix whose form is given in Eq.(10) for a truncation order $H=2$.

\section{Appendix 3: Derivation of the real Hill matrix}

It exists some situations where the presence of the pure imaginary number " $i$ " in the complex Hill matrix is a problem. An alternative is to deal with a real Hill matrix, although the formalism is more complicated. The difference with Appendix 2 is simply to expand the time-periodic equation of motion Eq. $(5), \dot{\mathbf{y}}(\tau)=$ $\mathbf{J}(\tau) \mathbf{y}(\tau)$, in real Fourier series.

In our particular case of a harmonic modulation, the $\bar{T}$-periodic Jacobian simply reads

$\mathbf{J}(\tau)=\frac{1}{2} \mathbf{J}_{c}^{0}+\mathbf{J}_{c}^{1} \cos (\beta \tau)$

where the expressions of $\mathbf{J}_{c}^{0}$ and $\mathbf{J}_{c}^{1}$ are given in Eq.(5). The $N=4$ Floquet forms $\mathbf{y}(\tau)$ given in the complex domain in Eq.(8) can be expressed by the real expansion

$\mathbf{y}(\tau)=\left(\frac{1}{2} \mathbf{a}^{0}+\sum_{h=1}^{\infty}\left[\mathbf{a}^{h} \cos (h \beta \tau)+\mathbf{b}^{h} \sin (h \beta \tau)\right]\right) e^{s \tau}$

and the associated time derivative reads

$$
\begin{aligned}
\dot{\mathbf{y}}(\tau) & =\left(\frac{1}{2} \mathbf{a}^{0} s+\sum_{h=1}^{\infty}\left[\left(s \mathbf{a}^{h}+h \beta \mathbf{b}^{h}\right) \cos (h \beta \tau)\right.\right. \\
& \left.\left.+\left(s \mathbf{b}^{h}-h \beta \mathbf{a}^{h}\right) \sin (h \beta \tau)\right]\right) e^{s \tau}
\end{aligned}
$$

Replacing the expanded expressions of $\mathbf{y}(\tau), \dot{\mathbf{y}}(\tau)$ and $\mathbf{J}(\tau)$ of Eq.(22)-(24) in Eq.(5), we can recast the equation of motion from the time domain to the spectral 
domain

$$
\begin{aligned}
\mathbf{0} & =\mathbf{J}(\tau) \mathbf{y}(\tau)-\dot{\mathbf{y}}(\tau) \\
\mathbf{0} & =\sum_{h=1}^{\infty}\left[\left(\frac{1}{2} \mathbf{J}_{c}^{0} \mathbf{a}^{h}-s \mathbf{a}^{h}-h \beta \mathbf{b}^{h}\right) \cos (h \beta \tau)\right] \\
& +\sum_{h=1}^{\infty}\left[\left(\frac{1}{2} \mathbf{J}_{c}^{0} \mathbf{b}^{h}-s \mathbf{b}^{h}+h \beta \mathbf{a}^{h}\right) \sin (h \beta \tau)\right] \\
& \left(\frac{1}{2} \mathbf{J}_{c}^{0}-s\right) \frac{\mathbf{a}^{0}}{2}+\mathbf{J}_{c}^{1}\left(\frac{\mathbf{a}_{0}}{2} \cos (\beta \tau)\right. \\
& +\sum_{h=1}^{\infty}\left[\frac{\mathbf{a}^{h}}{2}(\cos ((1+h) \beta \tau)+\cos ((1-h) \beta \tau))\right. \\
& \left.\left.+\frac{\mathbf{b}^{h}}{2}(\sin ((1+h) \beta \tau)-\sin ((1-h) \beta \tau))\right]\right)
\end{aligned}
$$

Like for Eq.(19) and (20), the harmonic balance method allows us to recast the problem in $2 H+1$ algebraic equations of dimension $N$ by independently equating to zero all the constant terms in $\cos (0 \beta \tau)$, the first harmonics $\cos (\beta \tau)$ and $\sin (\beta \tau)$, the second harmonics $\cos (2 \beta \tau)$ and $\sin (2 \beta \tau)$ and so on. By putting those equations in a matrix form, we obtain an eigenvalue problem $\left(\mathbf{H}^{H}-s \mathbf{1}\right) \mathbf{q}^{H}=\mathbf{0}$ where $\mathbf{H}^{H}$ is the $N \times(2 H+1)$-dimensional square real Hill matrix truncated at order $H, s$ and $\mathbf{q}^{H}$ are the $N \times(2 H+1)$ complex eigenvalues and eigenvectors of $\mathbf{H}^{H}$, respectively. If we order the eigenvector in the form $\mathbf{q}^{H}=$ $\left\{\frac{1}{2} \mathbf{a}^{0} \mathbf{a}^{1} \ldots \mathbf{a}^{H} \mathbf{b}^{1} \ldots \mathbf{b}^{H}\right\}^{T}$, the real Hill matrix reads, e.g. for $\mathbf{H}^{3}$ and $\mathbf{q}^{3}=\left\{\frac{1}{2} \mathbf{a}^{0} \mathbf{a}^{1} \mathbf{a}^{2} \mathbf{a}^{3} \mathbf{b}^{1} \mathbf{b}^{2} \mathbf{b}^{3}\right\}^{T}$ :

$$
\mathbf{H}^{3}=\left[\begin{array}{ccccccc}
\frac{1}{2} \mathbf{J}_{c}^{0} \frac{1}{2} \mathbf{J}_{c}^{1} & \mathbf{0}_{4} & \mathbf{0}_{4} & \mathbf{0}_{4} & \mathbf{0}_{4} & \mathbf{0}_{4} \\
\mathbf{J}_{c}^{1} & \frac{1}{2} \mathbf{J}_{c}^{0} & \frac{1}{2} \mathbf{J}_{c}^{1} & \mathbf{0}_{4} & -\beta \mathbf{1}_{4} & \mathbf{0}_{4} & \mathbf{0}_{4} \\
\mathbf{0}_{4} & \frac{1}{2} \mathbf{J}_{c}^{1} & \frac{1}{2} \mathbf{J}_{c}^{0} & \frac{1}{2} \mathbf{J}_{c}^{1} & \mathbf{0}_{4} & -2 \beta \mathbf{1}_{4} & \mathbf{0}_{4} \\
\mathbf{0}_{4} & \mathbf{0}_{4} & \frac{1}{2} \mathbf{J}_{c}^{1} & \frac{1}{2} \mathbf{J}_{c}^{0} & \mathbf{0}_{4} & \mathbf{0}_{4} & -3 \beta \mathbf{1}_{4} \\
\mathbf{0}_{4} & \beta \mathbf{1}_{4} & \mathbf{0}_{4} & \mathbf{0}_{4} & \frac{1}{2} \mathbf{J}_{c}^{0} & \frac{1}{2} \mathbf{J}_{c}^{1} & \mathbf{0}_{4} \\
\mathbf{0}_{4} & \mathbf{0}_{4} & 2 \beta \mathbf{1}_{4} & \mathbf{0}_{4} & \frac{1}{2} \mathbf{J}_{c}^{1} & \frac{1}{2} \mathbf{J}_{c}^{0} & \frac{1}{2} \mathbf{J}_{c}^{1} \\
\mathbf{0}_{4} & \mathbf{0}_{4} & \mathbf{0}_{4} & 3 \beta \mathbf{1}_{4} & \mathbf{0}_{4} & \frac{1}{2} \mathbf{J}_{c}^{1} & \frac{1}{2} \mathbf{J}_{c}^{0}
\end{array}\right]
$$

When replacing the eigenvalues $s$ and associated eigenvectors $\mathbf{q}$ of the real Hill matrix in the real Fourier expansion of the fundamental solutions given in Eq.(23), one gets a numerical approximation of the FFs of the systems. But like in the complex domain, because of truncation errors, the $N=4$ families of $(2 H+1)$ computed solutions $\mathbf{y}(\tau)$ do not all converge to the FFs and a sorting algorithm is needed. The computed eigenvalues are the same whether it comes from the real or complex Hill matrix. Consequently, the eigenvalue algorithm still holds with the real Hill matrix: by keeping the $N$ eigenvalues inside the spectral primitive cell $-\beta / 2 \leq \Im\left(s_{l}\right)<\beta / 2$, the latter (with their associated eigenvectors) will eventually lead to $N$ converged FFs as $H$ is increased. The eigenvector sorting algorithm that allows to compute the $N$ fundamental FFs also holds with the real Hill matrix. It still consists in computing the weighted means $w_{l}=\sum_{h}\left|\mathbf{p}_{l}^{h}\right| / \sum_{h}\left|\mathbf{p}_{l}^{h}\right|$ for $-H \leq h \leq H$ and keeping the spectrum associated with the $N$ weighted means that belong to the primitive cell $-1 / 2 \leq w_{l}<1 / 2$. The only supplementary step to compute $w_{l}$ with the real Hill matrix is that we need the complex/real transformation formulas $\mathbf{p}^{0}=\mathbf{a}^{0} / 2$ for $h=0$ and $\mathbf{p}^{-h}=\left(\mathbf{a}^{h}+i \mathbf{b}^{h}\right) / 2$ or $\mathbf{p}^{h}=\left(\mathbf{a}^{h}-i \mathbf{b}^{h}\right) / 2$ for $h>0$.

In the general case of a periodic modulation, the $\bar{T}$-periodic Jacobian would read

$\mathbf{J}(\tau)=\frac{1}{2} \mathbf{J}_{c}^{0}+\sum_{h=1}^{\infty}\left[\mathbf{J}_{c}^{h} \cos (h \beta \tau)+\mathbf{J}_{s}^{h} \sin (h \beta \tau)\right]$

In this situation, the linear equation of motion $\dot{\mathbf{y}}(\tau)-$ $\mathbf{J}(\tau) \mathbf{y}(\tau)=\mathbf{0}$, expanded in the real spectral domain, becomes much more complicated. However, the harmonic balance method can still be applied, leading to an eigenvalue problem $\left(\mathbf{H}^{H}-s \mathbf{1}\right) \mathbf{q}^{H}=\mathbf{0}$. The expression of the real Hill matrix $\mathbf{H}^{H}$ is yet more complicated than the one of Eq.(26) in the harmonic modulation case. If decomposed in a sum of block matrices and by ordering the eigenvector in the form $\mathbf{q}^{H}=$ $\left\{\frac{1}{2} \mathbf{a}^{0} \mathbf{a}^{1} \ldots \mathbf{a}^{H} \mathbf{b}^{1} \ldots \mathbf{b}^{H}\right\}^{T}, \mathbf{H}^{H}$ reads:

$\mathbf{H}^{H}=\left[\begin{array}{ccc}\frac{1}{2} \mathbf{J}_{c}^{0} & \frac{1}{2} \mathbf{J}_{c} & \frac{1}{2} \mathbf{J}_{s} \\ \mathbf{J}_{c}^{T} & {\left[\mathbf{K}_{c}+\mathbf{T}_{c}\right]} & {\left[\mathbf{K}_{s}-\mathbf{T}_{s}\right]} \\ \mathbf{J}_{s}^{T} & {\left[\mathbf{K}_{s}+\mathbf{T}_{s}\right]} & {\left[\mathbf{T}_{c}-\mathbf{K}_{c}\right]}\end{array}\right]$

where

$\mathbf{J}_{c}=\left\{\mathbf{J}_{c}^{1} \mathbf{J}_{c}^{2} \ldots \mathbf{J}_{c}^{H}\right\} \quad$ and $\quad \mathbf{J}_{s}=\left\{\mathbf{J}_{s}^{1} \mathbf{J}_{s}^{2} \ldots \mathbf{J}_{s}^{H}\right\}$

are $(N \times H N)$-dimensional block vectors $\left(\mathbf{J}_{c}^{T}\right.$ and $\mathbf{J}_{s}^{T}$ are the transpose of the above block vectors, not of the full matrices $\mathbf{J}_{c}$ and $\mathbf{J}_{s}$ meaning one has to be careful to not transpose the matrices $\mathbf{J}_{c}^{h}$ and $\mathbf{J}_{s}^{h}$ in the process but simply ordering them in a column block vector),

$\mathbf{K}_{c, s}=\frac{1}{2}\left[\begin{array}{ccccc}\mathbf{J}_{c, s}^{2} & \mathbf{J}_{c, s}^{3} & \mathbf{J}_{c, s}^{4} & \ldots & \mathbf{J}_{c, s}^{H+1} \\ \mathbf{J}_{c, s}^{3} & \mathbf{J}_{c, s}^{4} & \mathbf{J}_{c, s}^{5} & \ldots & \mathbf{J}_{c, s}^{H+2} \\ \mathbf{J}_{c, s}^{4} & \mathbf{J}_{c, s}^{5} & \mathbf{J}_{c, s}^{6} & \ldots & \mathbf{J}_{c, s}^{H+3} \\ \vdots & \vdots & \vdots & \ddots & \vdots \\ \mathbf{J}_{c, s}^{H+1} & \mathbf{J}_{c, s}^{H+2} & \mathbf{J}_{c, s}^{H+3} & \ldots & \mathbf{J}_{c, s}^{2 H}\end{array}\right]$

are $(N H \times N H)$-dimensional block matrices with harmonic contributions of the Jacobian either on cosine or 
sine, and where

$\mathbf{T}_{c}=\frac{1}{2}\left[\begin{array}{ccccc}\mathbf{J}_{c}^{0} & \mathbf{J}_{c}^{1} & \mathbf{J}_{c}^{2} & \ldots & \mathbf{J}_{c}^{H-1} \\ \mathbf{J}_{c}^{1} & \mathbf{J}_{c}^{0} & \mathbf{J}_{c}^{1} & \ldots & \mathbf{J}_{c}^{H-2} \\ \mathbf{J}_{c}^{2} & \mathbf{J}_{c}^{1} & \mathbf{J}_{c}^{0} & \ldots & \mathbf{J}_{c}^{H-3} \\ \vdots & \vdots & \vdots & \ddots & \vdots \\ \mathbf{J}_{c}^{H-1} & \mathbf{J}_{c}^{H-2} & \mathbf{J}_{c}^{H-3} & \ldots & \mathbf{J}_{c}^{0}\end{array}\right]$

and

$\mathbf{T}_{s}=\frac{1}{2}\left[\begin{array}{ccccc}2 \beta \mathbf{1}_{4} & -\mathbf{J}_{s}^{1} & -\mathbf{J}_{s}^{2} & \ldots & -\mathbf{J}_{s}^{H-1} \\ \mathbf{J}_{s}^{1} & 4 \beta \mathbf{1}_{4} & -\mathbf{J}_{s}^{1} & \ldots & -\mathbf{J}_{s}^{H-2} \\ \mathbf{J}_{s}^{2} & \mathbf{J}_{s}^{1} & 6 \beta \mathbf{1}_{4} & \ldots & -\mathbf{J}_{s}^{H-3} \\ \vdots & \vdots & \vdots & \ddots & \vdots \\ \mathbf{J}_{s}^{H-1} & \mathbf{J}_{s}^{H-2} & \mathbf{J}_{s}^{H-3} & \ldots & 2 H \beta \mathbf{1}_{4}\end{array}\right]$

are $(N H \times N H)$-dimensional block matrices. Although seemingly complicated if compared to the general complex Hill matrix given in Eq.(10) that is the sum of a complex block diagonal matrix and a real Toeplitz block matrix, the general real Hill matrix is relatively easy to numerically implement. Indeed, it is composed of $\mathbf{K}_{c}$ and $\mathbf{K}_{s}$ which are Hankel block matrices, $\mathbf{T}_{c}$ that is a Toeplitz matrix and $\mathbf{T}_{s}$ that is the sum of a real block diagonal matrix and a Toeplitz matrix. Applying the
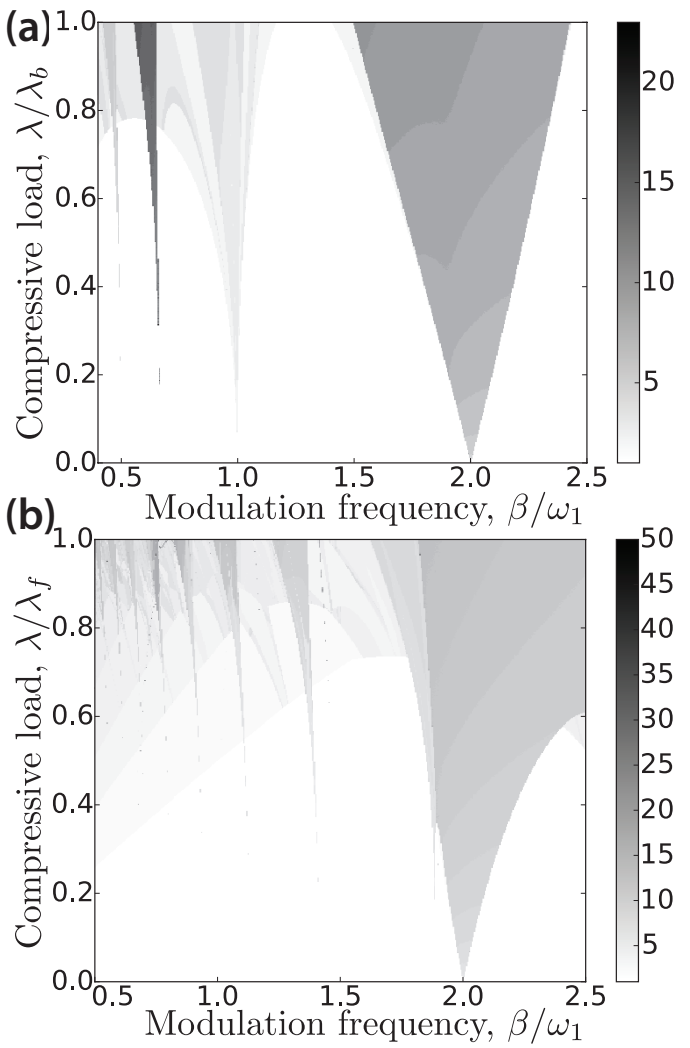

Fig. 14 Optimal spectral truncation order map $H_{\text {conv }}$ in the $\beta, \lambda$ space to ensure convergence. (a) Conservative case $\eta=0$. (b) Non conservative scenario $\eta=1$. eigenvector sorting algorithm on the real Hill matrix $\mathbf{H}^{H}$ of Eq.(28) allows to compute the $N$ fundamental FFs of a system in a general periodic state.

\section{Appendix 4: Spectral convergence of the stabil- ity charts}

The stability charts of Figs. 7 and 13 have been computed and validated with a classic monodromy matrix algorithm in the time domain [30]. For each parameter $(\beta, \lambda)$, the $N=4$ Floquet multipliers of the Monodromy matrix were computed with a sufficiently small time step to ensure convergence and served as a reference solution. The Hill matrix was then constructed for various increasing truncation order $H$. For each $H$, the Floquet multipliers $\rho_{n}^{\prime}$ where obtained from the $N=4$ fundamental Floquet exponents $s_{n}$ computed with the eigenvector sorting algorithm explained in Section 2.2, thanks to the relation $\rho_{n}^{\prime}=e^{s_{n} \bar{T}}$ where $\bar{T}=2 \pi / \beta$ is the dimensionless period of the considered perturbed elastic state. We defined a converged spectral truncation order $H_{\text {conv }}$ as the minimal $H$ for which the $N=4$ differences $\left|\rho_{n}^{\prime}-\rho_{n}\right|$ were not exceeding $1 \times 10^{-6}$. The map of the converged truncation order $H_{\text {conv }}$ in the $(\beta, \lambda)$ space is given in Figs.14(a) and (b) for $\eta=0$ and $\eta=1$, respectively.

The number of required harmonics $H_{c o n v}$ is generally larger as the modulation amplitude $\lambda$ is enhanced and the frequency modulation $\beta$ is decreased. Also more harmonics are required in the instability regions than in the stable ones. Those converged truncation order maps are the optimal ones when using the eigenvector sorting algorithm. If no sorting, the stability map would not converge and if using the eigenvalue sorting algorithm, one could need higher truncation order $H_{\text {conv }}$, especially for small $\beta$ or in the non conservative case. Note finally that it appears from Fig. 14 that more harmonics are needed in the non conservative case than in the conservative one. This trend is however exagerated as most of the numerical data converge for less than $H=25$ and only a very thin region, located around $\beta / \omega_{1} \approx 0.75$ and corresponding to a $2 \bar{T}$ instability of the second FF, needs $H=50$.

\section{References}

1. Adou, A.E., Tuckerman, L.S.: Faraday instability on a sphere: Floquet analysis. J. Fluid Mech. 805, 591-610 (2016)

2. Bekhoucha, F., Rechak, S., Duigou, L., Cadou, J.M.: Nonlinear forced vibrations of rotating anisotropic beams. Nonlinear Dyn. 74, 1281-1296 (2013) 
3. Bigoni, D., Noselli, G.: Experimental evidence of flutter and divergence instabilities induced by dry friction. J. Mech. Phys. Solids. 59, 2208-2226 (2011)

4. Bolotin, V.V.: The dynamic stability of elastic systems. Holden-Day, Inc. (1964)

5. Butcher, E.A., Sinha, S.C.: Normal forms and the structure of resonance sets in nonlinear time-periodic systems. Nonlinear Dyn. 23, 35-55 (2000)

6. Curtis, C., Deconinck, B.: On the convergence of Hill's method. Math. Comp. 79, 169-187 (2010)

7. Deconinck, B., Nathan Kutz J.: Computing spectra of linear operators using the Floquet-Fourier-Hill method. J. Comput. Phys. 219, 296-321 (2006)

8. Deconinck, B., Kiyak, F., Carter, J.D., Nathan Kutz J.: SpectrUW: A laboratory for the numerical exploration of spectra of linear operators. Math. Comput. Simul. 74, 370378 (2007)

9. Detroux, T., Renson, L., Masset, L., Kerschen, G.: The harmonic balance method for bifurcation analysis of largescale nonlinear mechanical systems. Comput. Methods Appl. Mech. Eng. 296, 18-38 (2015)

10. Ewins, D.J.: Modal testing: theory and practice. Research studies press Letchworth (1984)

11. Floquet, G.: Sur la théorie des équations différentielles. Annales scientifiques de l'École Normale Supérieure 8, 3$132(1879)$

12. Genta, G.: Dynamics of rotating systems. Springer Science \& Business Media. (2007)

13. Géradin, M., Rixen, D.: Mechanical vibrations: Theory and application to structural dynamics. John Wiley \& Sons, Inc. (1997)

14. Guckenheimer, J., Holmes, P.: Nonlinear oscillations, dynamical systems, and bifurcation of vector fields. New York Springer Verlag (1983)

15. Han, D.J.: Vibration analysis of periodically time-varying rotor system with transverse crack. Mech. Syst. Signal Process. 21, 28572879 (2007)

16. Han, Q.: Parametric instability of a Jeffcott rotor with rotationally asymmetric inertia and transverse crack. Nonlinear Dyn. 73, 827-842 (2013)

17. Hill, G.W.: On the part of the motion of the lunar perigee which is a function of the mean motions of the sun and moon. Acta Math. 8, 3-36 (1886)

18. Liu, J., Bertoldi, K.: Bloch wave approach for the analysis of sequential bifurcations in bilayer structures. Proc. R. Soc. A 471, 20150493 (2015)

19. Kumar, K., Tuckerman, L.S.: Parametric instability of the interface between two fluids. J. Fluid Mech. 279, 49-68 (1994)

20. Lazarus, A., Prabel, B., Combescure D.: A 3D finite element model for the vibration analysis of asymmetric rotating machines. J. Sound Vib. 329, 3780-3797 (2010)

21. Lazarus, A., de Langre, E., Manneville, P., Vincent, P., Perisanu, S., Ayari, A., Purcell, S.: Statics and dynamics of a nanowire in field emission. Int. J. Mech. Sci. 52(11), 13961406 (2010)

22. Lazarus, A., Thomas, O.: A harmonic-based method for computing the stability of periodic solutions of dynamical systems. Comp. Rend. Acad. Sci.: Mecanique 338, 510-517 (2010)

23. Lazarus, A., Thomas, O., Deü J.F.: Finite element reduced order models for nonlinear vibrations of piezoelectric layered beams with applications to NEMS. Finite Elem. Anal. Des. 49, 35-51 (2012)

24. Lee, C.W., Han, D.J., Suh, J.H., Hong, S.W.: Modal analysis of periodically time-varying linear rotor systems. J. Sound Vib. 303, 553-574 (2007)
25. McEwan, M.I., Wright, J.R., Cooper, J.E., Leung, A.Y.T.: A combined modal/finite element analysis technique for the dynamic response of a non-linear beam to harmonic excitation, J. Sound Vib. 243 , 601624 (2001)

26. Monteil, M., Touzé, C., Thomas, O. Benacchio, S.: Nonlinear forced vibrations of thin structures with tuned eigenfrequencies: the cases of 1:2:4 and 1:2:2 internal resonances, Nonlinear Dyn. 75, 175-200 (2014)

27. Moore, G.: Floquet theory as a computational tool, SIAM J. Numer. Anal. 42, 2522-2568 (2005)

28. Morse, P.M.: Vibration and sound. McGraw-Hill, NewYork (1948)

29. Nayfeh, A.H., Pai, P.F.: Linear and nonlinear structural mechanics. John Wiley \& Sons (2008)

30. Nayfeh, A.H., Mook, D.T.: Nonlinear oscillations. John Wiley \& Sons (2008)

31. Païdoussis, M.P., Price, S.J., De Langre E.: Fluidstructure interactions: cross-flow-induced instabilities. Cambridge University Press (2010)

32. Pandiyan, R., Sinha, S.C.: Analysis of time-periodic nonlinear dynamical systems undergoing bifurcations. Nonlinear Dyn. 8, 21-43 (1995)

33. Peeters, M., Viguié, R., Sérandour, G., Kerschen, G., Golinval, J.-C.: Nonlinear normal modes, Part II: Toward a practical computation using numerical continuation techniques, Mech. Syst. Signal Process. 23, 195-216 (2009)

34. Peletan, L., Baguet, S., Torkhani, M., Jacquet-Richardet, G.: A comparison of stability computational methods for periodic solution of nonlinear problems with application to rotor dynamics. Nonlinear Dyn. 72, 1-12 (2013)

35. Poincaré, H.: Sur les déterminants d'ordre infini. Bulletin de la S. M. F. 14, 77-90 (1886)

36. Shimanouchi, T.: Tables of Molecular Vibrational Frequencies Part 5. J. Phys. Chem. Ref. Data, 1, 189-216 (1972)

37. Sinha, S.C., Wu, D.-H.: An efficient computational scheme for the analysis of periodic systems. J. Sound Vib., 151, 91-117 (1991)

38. Sinha, S.C., Pandiyan, R., Bibb, J.S.: Liapunov-Floquet Transformation: Computation and Applications to Periodic Systems. J. Vib. Acoust., 118, 209-219 (1996)

39. Sinha, S.C., Butcher, E.A., David, A.: Construction of dynamically equivalent time-invariant forms for timeperiodic systems. Nonlinear Dyn. 16, 203-221 (1998)

40. Sinha, S.C., Redkar, S., Deshmukh, V., Butcher, E.A.: Order reduction of parametrically excited nonlinear systems: techniques and applications. Nonlinear Dyn. 41, 237$273(2005)$

41. Thomas, O., Mathieu, F., Mansfield, W., HUang, C., Trolier-McKinstry, S. Nicu, L: Efficient parametric amplification in micro-resonators with integrated piezoelectric actuation and sensing capabilities. Appl. Phys. Lett. 102, 163504 (2013)

42. Tommasini, M., Kirillov, O.N., Misseroni, D., Bigoni, D.: The destabilizing effect of external damping: Singular flutter boundary for the Pflüger column with vanishing external dissipation. J. Mech. Phys. Solids. 91, 204-215 (2016)

43. Wang, X., Hale, J.K.: On monodromy matrix computation. Comput. Methods Appl. Mech. Eng., 190, 2263-2275 (2001)

44. Whittaker, E.T., Watson, G.N.: A course of modern analysis, Cambridge university press (1927)

45. Zhou, J., Hagiwara, T., Araki, M.: Spectral analysis and eigenvalues computation of the harmonic state operators in continuous-time periodic systems. Syst. Control Lett. 53, 141155 (2004) 